\title{
Knot commensurability and the Berge conjecture
}

\author{
Michel Boileau \\ STEVEN BOYER \\ RADU CEBANU \\ GeneVIEVE S WALSH
}

\begin{abstract}
We investigate commensurability classes of hyperbolic knot complements in the generic case of knots without hidden symmetries. We show that such knot complements which are commensurable are cyclically commensurable, and that there are at most 3 hyperbolic knot complements in a cyclic commensurability class. Moreover if two hyperbolic knots have cyclically commensurable complements, then they are fibred with the same genus and are chiral. A characterization of cyclic commensurability classes of complements of periodic knots is also given. In the nonperiodic case, we reduce the characterization of cyclic commensurability classes to a generalization of the Berge conjecture.
\end{abstract}

$57 \mathrm{M} 10,57 \mathrm{M} 25$

\section{Introduction}

We work in the oriented category throughout this paper. In particular we endow the complement of any knot $K \subset S^{3}$ with the orientation inherited from the standard orientation on $S^{3}$. We consider two knots to be equivalent if there is an orientationpreserving homeomorphism of $S^{3}$ taking one to the other. Covering maps will be assumed to preserve orientation unless stated otherwise.

Two oriented orbifolds are commensurable if they have homeomorphic finite sheeted covers. We are interested in studying commensurability classes of knot complements in $S^{3}$. By abuse of language we will say that two knots in the 3 -sphere are commensurable if their complements are commensurable. Set

$$
\mathcal{C}(K)=\left\{\text { knots } K^{\prime} \subset S^{3}: K^{\prime} \text { is commensurable with } K\right\} .
$$

A difficult and widely open problem is to describe commensurability classes of knots.

One of our main concerns is to provide a priori bounds on the number of hyperbolic knots in a given commensurability class. Unless otherwise stated, knots are considered 
to be in $S^{3}$. Hence in this paper $K \subset S^{3}$ will be a hyperbolic knot. Its complement $S^{3} \backslash K=\mathbf{H}^{3} / \Gamma_{K}$ is a complete, oriented, hyperbolic 3-manifold of finite volume, where $\pi_{1}\left(S^{3} \backslash K\right) \cong \Gamma_{K} \subset \operatorname{PSL}(2, \mathbb{C})=\operatorname{Isom}^{+}\left(\mathbf{H}^{3}\right)$ is a lattice. Any knot $K^{\prime}$ commensurable with $K$ is also hyperbolic and the commensurability condition is equivalent to the fact that $\Gamma_{K}$ and some conjugate of $\Gamma_{K^{\prime}}$ in $\operatorname{Isom}^{+}\left(\mathbf{H}^{3}\right)$ have a common finite index subgroup.

Recall that the commensurator of a group $\Gamma$ in $\operatorname{PSL}(2, \mathbb{C})$ is

$$
C^{+}(\Gamma)=\left\{g \in \operatorname{PSL}(2, \mathbb{C}):\left[\Gamma: \Gamma \cap g^{-1} \Gamma g\right]<\infty \text { and }\left[g^{-1} \Gamma g: \Gamma \cap g^{-1} \Gamma g\right]<\infty\right\} .
$$

Then $K$ and $K^{\prime}$ are commensurable if and only if $C^{+}\left(\Gamma_{K}\right)$ and $C^{+}\left(\Gamma_{K^{\prime}}\right)$ are conjugate in $\operatorname{PSL}(2, \mathbb{C})$. An element $g \in C^{+}\left(\Gamma_{K}\right)$ induces an orientation-preserving isometry between two finite sheeted coverings of $S^{3} \backslash K$. It is called a hidden symmetry of $K$ if it is not the lift of an isometry of $S^{3} \backslash K$.

The group of isotopy classes of orientation-preserving symmetries of $\left(S^{3}, K\right)$ is isomorphic, in the obvious way, to $\operatorname{Isom}^{+}\left(S^{3} \backslash K\right)$, the group of orientation-preserving isometries of $S^{3} \backslash K$. It is also isomorphic to the quotient group $N^{+}(K) / \Gamma_{K}$, where $N^{+}(K)$ is the normalizer of $\Gamma_{K}$ in $\operatorname{PSL}(2, \mathbb{C})$. We will use either description as convenient. Then $K$ has hidden symmetries if and only if $N^{+}(K)$ is strictly smaller than $C^{+}\left(\Gamma_{K}\right)$. Hyperbolic knots with hidden symmetries appear to be rare, as Neumann and Reid [29] showed that if $K$ has hidden symmetries then the cusp shape of $\mathbf{H}^{3} / \Gamma_{K}$ is contained in $\mathbb{Q}[i]$ or $\mathbb{Q}[\sqrt{-3}]$.

Currently, the only knots known to admit hidden symmetries are the figure- 8 and the two dodecahedral knots of Aitchison and Rubinstein described in [1] (cf Conjecture 1.3 below). These three knots have cusp field $\mathbb{Q}[\sqrt{-3}]$. There is one known example of a knot with cusp field $\mathbb{Q}[i]$, and it does not admit hidden symmetries. See Boyd's notes [7, page 17] and Goodman, Heard and Hodgson [17].

It is a fundamental result of Margulis that a finite covolume, discrete subgroup $\Gamma$ of $\operatorname{PSL}(2, \mathbb{C})$ is nonarithmetic if and only if there is a unique minimal orbifold in the commensurability class of $\mathbf{H}^{3} / \Gamma$, namely $\mathbf{H}^{3} / C^{+}(\Gamma)$. Reid [39] has shown that the figure-8 is the only arithmetic knot (ie knot with arithmetic complement) in $S^{3}$, hence it is the unique knot in its commensurability class. So in what follows we only consider nonarithmetic knots. In particular, $C^{+}\left(\Gamma_{K}\right)$ is a lattice in $\operatorname{PSL}(2, \mathbb{C})$ and the unique minimal element in the commensurability class of $S^{3} \backslash K=\mathbf{H}^{3} / \Gamma_{K}$ is the oriented orbifold $\mathbf{H}^{3} / C^{+}\left(\Gamma_{K}\right)$, which we denote by $\mathcal{O}_{\min }(K)$.

When $K$ has no hidden symmetries,

$$
\mathcal{O}_{\min }(K)=\mathbf{H}^{3} / N^{+}(K)=\left(S^{3} \backslash K\right) / \operatorname{Isom}^{+}\left(S^{3} \backslash K\right) .
$$


The positive solution of the Smith conjecture implies that $\operatorname{Isom}^{+}\left(S^{3} \backslash K\right)$ is cyclic or dihedral and the subgroup of $\operatorname{Isom}^{+}\left(S^{3} \backslash K\right)$ which acts freely on $K$ is cyclic of index at most 2 . We denote this subgroup by $Z(K)$. Clearly the oriented orbifold

$$
\mathcal{Z}_{K}=\left(S^{3} \backslash K\right) / Z(K)
$$

has a torus cusp and either coincides with the minimal element in the commensurability class of $S^{3} \backslash K$ or is a 2 -fold cover of it. Hence in this case the cusp of $\mathcal{O}_{\min }(K)$ is flexible: its horospherical cross-section is either $T^{2}$ or $S^{2}(2,2,2,2)$. Neumann and Reid [29] proved that a nonarithmetic knot $K$ has no hidden symmetries if and only if $\mathcal{O}_{\min }(K)$ has a flexible cusp and further, that this condition is equivalent to the fact that $S^{3} \backslash K$ normally covers $\mathcal{O}_{\min }(K)$. If a commensurability class has a unique minimal element with a single cusp and the cusp is flexible, we call the commensurability class itself flexible. When $K$ does admit hidden symmetries, the horospherical crosssection of $\mathcal{O}_{\min }(K)$ is a Euclidean turnover, which is rigid. If a commensurability class has a unique minimal element with a single cusp which is rigid, we say that the commensurability class itself is rigid.

Reid and Walsh [40] proved that a hyperbolic 2-bridge knot is unique in its commensurability class and raised the following conjecture:

Conjecture 1.1 (Reid-Walsh [40]) For a hyperbolic knot $K \subset S^{3},|\mathcal{C}(K)| \leq 3$.

The commensurability class of the $(-2,3,7)$ pretzel knot is flexible (see Macasieb and Mattman [26]) and contains exactly three knots. Hoffman [21] has constructed an infinite family of hyperbolic knots with this property.

Our first result proves the conjecture in the generic case:

Theorem 1.2 A flexible commensurability class contains at most three hyperbolic knot complements.

A precise formulation of the expected genericity of the flexible case is contained in the following conjecture of Neumann and Reid:

Conjecture 1.3 (Neumann-Reid) The only rigid commensurability class containing hyperbolic knot complements is the commensurability class of the dodecahedral knots, and there are only two knot complements in this class.

See also Neumann [28, Theorem 2].

We say that two hyperbolic orbifolds are cyclically commensurable if they have a common finite cyclic cover. We denote by $\mathcal{C C}(K)$ the set of hyperbolic knots cyclically 
commensurable with $K$. A priori, cyclic commensurability is much more restrictive than commensurability. However for hyperbolic knots without hidden symmetries, the commensurability class and the cyclic commensurability class coincide. Theorem 1.2 follows immediately from the following results:

Theorem 1.4 (1) Knots without hidden symmetries which are commensurable are cyclically commensurable.

(2) A cyclic commensurability class contains at most three hyperbolic knot complements.

In this article we analyze the case of hyperbolic knots which are commensurable to other hyperbolic knots and which do not admit hidden symmetries. However, many of our results hold for any hyperbolic knots with hidden symmetries which are cyclically commensurable to other knots. This conjecturally does not happen (see Conjecture 4.14).

Geometrization combines with the work of González-Acuña and Whitten [16] to determine close connections between the family of knots which are cyclically commensurable to other knots and the family of knots which admit lens space surgeries: if the complement of a knot $K$ is covered by another knot complement, then the covering is cyclic and this occurs if and only if $K$ admits a nontrivial lens space surgery. In this situation, a fundamental result of $\mathrm{Ni}$ [30] implies that $K$ is fibred. Here we show that distinct knots without hidden symmetries which are commensurable are obtained from primitive knots in orbi-lens spaces (Section 3) which admit nontrivial orbi-lens space surgeries. Further, we prove an analogue of Ni's result in the orbifold setting:

Theorem 1.5 Let $K$ be a knot in an orbi-lens space $\mathcal{L}$ which is primitive in $\mathcal{L}$. If $K$ admits a nontrivial orbi-lens space surgery, then the exterior of $K$ admits a fibring by 2-orbifolds with base the circle.

Our next result is an interesting by-product of the method of proof of Theorem 1.5. For the definition of a 1-bridge braid in a solid torus, refer to Section 5.

Proposition 1.6 Let $M$ be the exterior of a hyperbolic 1-bridge braid in a solid torus $V$. Then each top-dimensional face of the Thurston norm ball in $H_{2}(M, \partial M ; \mathbb{R})$ is a fibred face. 
Theorem 1.7 Let $K$ be a hyperbolic knot. If $|\mathcal{C C}(K)| \geq 2$ then:

(1) $K$ is a fibred knot.

(2) the genus of $K$ is the same as that of any $K^{\prime} \in \mathcal{C C}(K)$.

(3) the volume of $K$ is different from that of any $K^{\prime} \in \mathcal{C C}(K) \backslash K$. In particular, the only mutant of $K$ contained in $\mathcal{C C}(K)$ is $K$.

(4) $K$ is chiral and not commensurable with its mirror image.

In particular this result holds for a hyperbolic knot $K$ without hidden symmetries and any $K^{\prime} \in \mathcal{C}(K) \backslash K$.

We pause to note the marked difference between the case of flexible and rigid commensurability classes containing knot complements. Recall that the commensurability class of the two dodecahedral knots [1] is the only known rigid commensurability class containing knot complements. These knots do not satisfy any of the conditions above: one dodecahedral knot is fibred, the other isn't; the knots have different genus; they have the same volume; the knots are both amphichiral [2, 12.1]. In addition, they are not cyclically commensurable, in contrast with Theorem 1.4.

A knot $K$ is periodic if it admits a nonfree symmetry with an axis disjoint from $K$. As a consequence of the works of Berge [4] and Gabai [13] we obtain the following characterisation of cyclic commensurability classes of periodic knots. Refer to Section 5 for the definitions of Berge-Gabai knots and unwrapped Berge-Gabai knots.

Theorem 1.8 Let $K$ be a periodic hyperbolic knot. If $|\mathcal{C C}(K)| \geq 2$ then:

(1) $K$ has a unique axis of symmetry disjoint from $K$.

(2) $K$ is obtained by unwrapping a Berge-Gabai knot $\bar{K}$ in an orbi-lens space. In particular $K$ is strongly invertible.

(3) each $K^{\prime} \in \mathcal{C C}(K)$ is determined by unwrapping the Berge-Gabai knot represented by the core of the surgery solid torus in an orbi-lens space obtained by Dehn surgery along $\bar{K}$.

In particular this result holds for a periodic hyperbolic knot $K$ without hidden symmetries and any $K^{\prime} \in \mathcal{C}(K)$.

The proof of Theorem 1.8 reduces the characterisation of hyperbolic knots $K \subset S^{3}$ such that $|\mathcal{C C}(K)| \geq 2$ to the case where $Z(K)$ acts freely on $S^{3}$ and to the construction of all primitive knots in a lens space with a nontrivial lens space surgery. It is a result of Bonahon and Otal [6] that for each $g \geq 1$, a lens space admits a unique genus $g$ Heegaard splitting, which is a stabilization of the genus 1 splitting. 
Problem 1.9 Characterize primitive knots $\bar{K}$ in a lens space $L$ which admit a nontrivial lens space surgery. In particular, is every such knot a doubly primitive knot on the genus 2 Heegaard surface of $L$ ?

Suppose $\bar{K}$ is in $S^{3}$. Then this problem is the setting of the Berge conjecture, which contends that a knot in $S^{3}$ which admits a nontrivial lens space surgery is doubly primitive. Doubly primitive knots are knots which lie on the genus 2 Heegaard surface in such a way that the knot represents a generator of the fundamental group of each handlebody.

A fundamental result of Schwartz [42] implies that the fundamental groups of two hyperbolic knots $K, K^{\prime}$ are quasi-isometric if and only if $K^{\prime}$ is commensurable with $K$ or with its mirror image. Proposition 5.8 below shows that a knot without hidden symmetries cannot be commensurable to its mirror image. Therefore, as a consequence of Theorems 1.4, 1.7 and 1.8 we obtain the following results on quasi-isometry classes of knot groups:

Corollary 1.10 Let $K$ be a hyperbolic knot without hidden symmetries. Then there are at most three knots $K^{\prime}$ with group $\pi_{1}\left(S^{3} \backslash K^{\prime}\right)$ quasi-isometric to $\pi_{1}\left(S^{3} \backslash K\right)$. Moreover $\pi_{1}\left(S^{3} \backslash K\right)$ is the unique knot group in its quasi-isometry class in the following cases:

(i) $K$ is not fibred.

(ii) $K$ is amphichiral.

(iii) $K$ is periodic and is not an unwrapped Berge-Gabai knot; for instance, $K$ is periodic but not strongly invertible.

(iv) $K$ is periodic with two distinct axes of symmetry.

The paper is organized as follows. Theorem 1.4 is proved in Section 4. Theorem 1.8 and (3) of Theorem 1.7 are contained in Section 5. Theorem 1.5, parts (1) and (2) of Theorem 1.7 and Proposition 1.6 are proven in Section 6. Part (4) of Theorem 1.7 is proven in Section 7. Sections 2 and 3 are devoted to conventions and background on certain spherical orbifolds.

Acknowledgements We thank Jake Rasmussen for explaining the proof that a knot in a lens space which admits a nontrivial lens space surgery has fibred complement. We also benefited from helpful conversations with Walter Neumann and Alan Reid. 


\section{Slopes, Dehn filling and cusp types}

A slope on a torus $T$ is an isotopy class of essential simple closed curves. The set of slopes on $T$ corresponds bijectively, in the obvious way, with \pm -classes of primitive elements of $H_{1}(T)$. Thus to each slope $r$ we associate the primitive classes $\pm \alpha(r) \in H_{1}(T)$ represented by a simple closed curve in the class of $r$. The distance between two slopes $r, r^{\prime}$ on $T$ is given by $\Delta\left(r, r^{\prime}\right)=\left|\alpha(r) \cdot \alpha\left(r^{\prime}\right)\right|$.

Given a slope $r$ on a torus boundary component $T$ of a 3-manifold $M$, the $r-D e h n$ filling of $M$ with slope $r$ is the 3-manifold $M(T ; r):=\left(S^{1} \times D^{2}\right) \cup_{f} M$ where $f$ is any homeomorphism $\partial\left(S^{1} \times D^{2}\right) \rightarrow T$ such that $f\left(\{*\} \times \partial D^{2}\right)$ represents $r$. It is well-known that $M(T ; r)$ is independent of the choice of $f$. When there is no risk of ambiguity, we shall usually denote $M(T ; r)$ by $M(r)$.

Recall that topologically, a cusp of a complete, finite volume, orientable, hyperbolic 3 -orbifold is of the form $\mathcal{B} \times \mathbb{R}$ where $\mathcal{B}$ is a closed, connected, orientable, Euclidean 2 -orbifold. In this case, we say that the cusp is a $\mathcal{B}$ cusp.

A slope $r$ in a torus cusp of a complete, noncompact, finite volume hyperbolic 3orbifold $\mathcal{O}$ is a cusp isotopy class of essential simple closed curves which lie on some torus section of the cusp. Inclusion induces a bijection between the slopes on a torus cross-section of the cusp with those in the cusp, and we identify these sets below.

When $M$ is a compact 3-manifold with torus boundary, the kernel of $i_{*}: H_{1}(\partial M) \rightarrow$ $H_{1}(M)$ has rank one [20, Lemma 3.5]. Thus there is a $\lambda \in H_{1}(\partial M)$ such that $\pm \lambda$ are the only primitive classes in $H_{1}(\partial M) \cong \pi_{1}(\partial M)$ which are trivial in $H_{1}(M ; \mathbb{Q})$. We call the class $\lambda \in H_{1}(\partial M)$ the rational longitude.

Lemma 2.1 Let $\mathcal{O}$ be a complete, finite volume, orientable, hyperbolic 3-orbifold which has one end, a torus cusp, and let $r$ be a slope in this cusp. Then for any orientation-preserving homeomorphism $f: \mathcal{O} \rightarrow \mathcal{O}$, the slope $f(r)$ equals $r$.

Proof Our assumptions imply that $|\mathcal{O}|$ is the interior of a compact, connected, orientable 3-manifold $M$ with torus boundary to which we can extend $f$. To prove the lemma it suffices to show that $f$ acts as multiplication by \pm 1 on $H_{1}(\partial M)$. First note that $f_{*}\left(\lambda_{M}\right)=\epsilon \lambda_{M}$ where $\epsilon \in\{ \pm 1\}$ and $\lambda_{M} \in H_{1}(\partial M)$ is the rational longitude of $M$.

Let $\rho: \pi_{1}(\mathcal{O}) \rightarrow \operatorname{PSL}(2, \mathbb{C})$ be a discrete faithful representation. By Mostow-Prasad rigidity, there is an element $A \in \operatorname{PSL}(2, \mathbb{C})$ such that $\rho \circ f_{\#}=A \rho A^{-1}$. In particular,

$$
\rho\left(\lambda_{M}\right)^{\epsilon}=\rho\left(f_{\#}\left(\lambda_{M}\right)\right)=A \rho\left(\lambda_{M}\right) A^{-1} .
$$


Without loss of generality we can assume that $\rho\left(\lambda_{M}\right)$ is upper triangular and parabolic. Then it is easy to verify that $A$ is upper triangular and parabolic when $\epsilon=1$ or upper triangular with diagonal entries $\pm i$ when $\epsilon=-1$. A simple calculation then shows that when $\epsilon=1, \rho\left(f_{\#}(\gamma)\right)=\rho(\gamma)$ for each $\gamma \in \pi_{1}(\partial M)$, which implies that $f_{*}$ is the identity. Similarly when $\epsilon=-1$ it's easy to see that $f_{*}=-I$.

Given two slopes $r, r^{\prime}$ in the cusp, the reader will verify that the distance between two of their representatives contained in some torus cross-section of the cusp is independent of the cross-section, and we define the distance between $r$ and $r^{\prime}$, denoted $\Delta\left(r, r^{\prime}\right)$, to be this number.

Let $r$ be a slope in a torus cusp of $\mathcal{O}$ and $\widehat{\mathcal{O}}$ an orbifold obtained by truncating $\mathcal{O}$ along the cusp. The Dehn filling of $\mathcal{O}$ of slope $r$, denoted $\mathcal{O}(r)$, is the $r$-Dehn filling of $\widehat{\mathcal{O}}$.

\section{Orbi-lens spaces}

We denote the singular set of an orbifold $\mathcal{O}$ by $\Sigma(\mathcal{O})$ throughout the paper.

An orbi-lens space is the quotient orbifold of $S^{3}$ by a finite cyclic subgroup of $\mathrm{SO}(4)$, the group of orientation preserving isometries of $S^{3}$. We begin by examining their structure.

The first homology group of an orbifold is the abelianisation of its fundamental group.

A knot in an orbi-lens space $\mathcal{L}$ is primitive if it carries a generator of $H_{1}(\mathcal{L})$.

Lemma 3.1 Let $Z$ be a finite cyclic subgroup of $\mathrm{SO}(4)$ of order $n$ and fix a generator $\psi$ of $Z$. There are a genus one Heegaard splitting $S^{3}=V_{1} \cup V_{2}$, cores $C_{1}, C_{2}$ of $V_{1}, V_{2}$, and integers $a_{1}, a_{2} \geq 1$ such that

(1) both $V_{1}$ and $V_{2}$ are $Z$-invariant.

(2) $\psi$ acts by rotation of order $a_{1}$ on $C_{1}$ and order $a_{2}$ on $C_{2}$. Moreover, the $Z$-isotropy subgroup of a point in

- $S^{3} \backslash\left(C_{1} \cup C_{2}\right)$ is trivial.

- $C_{1}$ is generated by $\psi^{a_{1}}$ and has order $\bar{a}_{2}=n / a_{1}$,

- $C_{2}$ is generated by $\psi^{a_{2}}$ and has order $\bar{a}_{1}=n / a_{2}$.

Therefore $n=\operatorname{lcm}\left(a_{1}, a_{2}\right), \bar{a}_{1}=a_{1} / \operatorname{gcd}\left(a_{1}, a_{2}\right), \bar{a}_{2}=a_{2} / \operatorname{gcd}\left(a_{1}, a_{2}\right)$, so $\operatorname{gcd}\left(\bar{a}_{1}, \bar{a}_{2}\right)=1$.

(3) $\left|S^{3} / Z\right|$ is the lens space with fundamental $\operatorname{group} \mathbb{Z} / \operatorname{gcd}\left(a_{1}, a_{2}\right)$ and genus one Heegaard splitting $\left(V_{1} / Z\right) \cup\left(V_{2} / Z\right)$. The ramification index of a point $y \in\left|S^{3} / Z\right|$ is $\bar{a}_{2}$ if $y \in C_{1} / Z, \bar{a}_{1}$ if $y \in C_{2} / Z$, and 1 otherwise. Hence $\Sigma\left(S^{3} / Z\right) \subseteq\left(C_{1} / Z\right) \cup\left(C_{2} / Z\right)$. 
Proof We can find two mutually orthogonal 2-dimensional subspaces of $\mathbb{R}^{4}$ on which $\psi$ acts by rotation. Thus if we think of these subspaces as the two coordinate planes of $\mathbb{C}^{2}, \psi$ has the form

$$
\psi(z, w)=\left(e^{2 \pi i \alpha_{1} / a_{1}} z, e^{2 \pi i \alpha_{2} / a_{2}} w\right)
$$

where $\operatorname{gcd}\left(\alpha_{1}, a_{1}\right)=\operatorname{gcd}\left(\alpha_{2}, a_{2}\right)=1$ and $n=\operatorname{lcm}\left(a_{1}, a_{2}\right)$. The subgroup of $Z$ which

- fixes $(z, w)$ with $z w \neq 0$ is the trivial subgroup.

- fixes the $z$-plane is generated by $\psi^{a_{1}}$ and has order $\bar{a}_{2}=n / a_{1}=a_{2} / \operatorname{gcd}\left(a_{1}, a_{2}\right)$.

- fixes the $w$-plane is generated by $\psi^{a_{2}}$ and has order $\bar{a}_{1}=n / a_{2}=a_{1} / \operatorname{gcd}\left(a_{1}, a_{2}\right)$.

The genus one Heegaard splitting of $S^{3}$ given by the two solid tori $V_{1}=\{(z, w)$ : $\left.|z|^{2}+|w|^{2}=1,|w| \leq 1 / \sqrt{2}\right\}$ and $V_{2}=\left\{(z, w):|z|^{2}+|w|^{2}=1,|z| \leq 1 / \sqrt{2}\right\}$ is invariant under $Z$ and determines a genus one Heegaard splitting of $\left|S^{3} / Z(K)\right|$. Further, the isotropy subgroup of a point $(z, w) \in S^{3}$ is trivial if $|z w| \neq 0, \mathbb{Z} / \bar{a}_{2}$ if $w=0$, and $\mathbb{Z} / \bar{a}_{1}$ if $z=0$. The conclusions of the lemma follow from these observations.

Corollary 3.2 A 3-orbifold $\mathcal{L}$ is an orbi-lens space if and only if $|\mathcal{L}|$ is a lens space which admits a genus one Heegaard splitting $|\mathcal{L}|=V_{1} \cup V_{2}$ such that $\Sigma(\mathcal{L})$ is a closed submanifold of the union of the cores $C_{1}, C_{2}$ of $V_{1}, V_{2}$, and there are coprime positive integers $b_{1}, b_{2} \geq 1$ such that a point of $C_{j}$ has isotropy group $\mathbb{Z} / b_{j}$. In the latter case, $\pi_{1}(\mathcal{L}) \cong \mathbb{Z} /\left(b_{1} b_{2}\left|\pi_{1}(|\mathcal{L}|)\right|\right)$.

Proof Lemma 3.1 shows that an orbi-lens space has the form claimed in the corollary. Conversely, suppose that $\mathcal{L}$ is a 3 -orbifold for which $|\mathcal{L}| \cong L(p, q)$ admits a genus one Heegaard splitting $|\mathcal{L}|=V_{1} \cup V_{2}$ such that $\Sigma(\mathcal{L})$ is a closed submanifold of the union of the cores $C_{1}, C_{2}$ of $V_{1}, V_{2}$, and there are coprime positive integers $b_{1}, b_{2} \geq 1$ such that a point of $C_{j}$ has isotropy group $\mathbb{Z} / b_{j}$. It is straightforward to verify that there is a $b_{1} b_{2} p$-fold cyclic cover $S^{3} \rightarrow \mathcal{L}$ whose deck transformations lie in $\mathrm{SO}(4)$. Thus $\mathcal{L}$ is an orbi-lens space.

We will use $\mathcal{L}\left(p, q ; b_{1}, b_{2}\right)$ to denote the orbifold described in the corollary. As we are mainly concerned with the case $b_{1}=1$ and $b_{2}=a$, we use $\mathcal{L}(p, q ; a)$ to denote $\mathcal{L}(p, q ; 1, a)$. When $a=1, \mathcal{L}(p, q ; a)$ is just $L(p, q)$. 


\section{Proof of Theorem 1.4}

Proposition 4.1 Two hyperbolic knot complements have a common finite-index cyclic cover if and only if they have a common finite-index cyclic quotient. Moreover, two cyclically commensurable hyperbolic knot complements have the same normalizers in $\operatorname{PSL}(2, \mathbb{C})$.

Proof The fact that a common finite-index cyclic quotient implies a common finiteindex cyclic cover is immediate from the isomorphism theorems. Consider the converse then.

Suppose that $S^{3} \backslash K_{1} \cong \mathbf{H}^{3} / \Gamma_{1}$ and $S^{3} \backslash K_{2} \cong \mathbf{H}^{3} / \Gamma_{2}$ have a common finite-index cyclic cover $M \cong \mathbf{H}^{3} / \Gamma_{M}$. We may assume, after conjugating, that $\Gamma_{M} \subseteq \Gamma_{1} \cap \Gamma_{2}$. Since knot complements have unique cyclic covers of a given order, each isometry of $S^{3} \backslash K_{j}$ is covered by an isometry of $M$ for $j=1,2$. Recall that the cyclic subgroup $Z(K) \subset$ Isom $^{+}\left(S^{3} \backslash K\right)$ acts freely on $K$ and that the orbifold $\mathcal{Z}_{K}=\left(S^{3} \backslash K\right) / Z(K)$ has a torus cusp. Let $\overline{Z\left(K_{1}\right)}$ be the subgroup of $\operatorname{Isom}^{+}(M)$ covering $Z\left(K_{1}\right)$ and define $\overline{Z\left(K_{2}\right)}$ similarly. By construction, $\overline{Z\left(K_{1}\right)}$ and $\overline{Z\left(K_{2}\right)}$ act freely in the cusp of $M$ and since this cusp is unique, the lift of each element of the subgroup $\overline{Z\left(K_{j}\right)}$ to $\mathbf{H}^{3}$ is parabolic $(j=1,2)$. Thus $\overline{Z\left(K_{1}\right)}$ and $\overline{Z\left(K_{2}\right)}$ act by Euclidean translations in each horotorus of the cusp of $M$. It follows that $\overline{Z\left(K_{1}\right)}$ and $\overline{Z\left(K_{2}\right)}$ generate an abelian subgroup $A$ of $\operatorname{Isom}^{+}(M)$ which acts by Euclidean translations in the horotori. The covering group of $M \rightarrow S^{3} \backslash K_{i}$ descends to $Z\left(K_{j}\right)$ since the two groups of covering transformations commute. Thus there are regular covers $S^{3} \backslash K_{j} \rightarrow \mathcal{O}=M / A$ for $j=1,2$ where $\mathcal{O}$ has a torus cusp. These covers are cyclic by [16; 39, Lemma 4] and so $S^{3} \backslash K_{1}$ and $S^{3} \backslash K_{2}$ have a common cyclic quotient, $M / A=\mathcal{Z}_{K_{1}}=\mathcal{Z}_{K_{2}}$. Note, moreover, that there is a cover $S^{3} \backslash K_{1} \rightarrow\left(S^{3} \backslash K_{2}\right) / \operatorname{Isom}^{+}\left(S^{3} \backslash K_{2}\right)=\mathbf{H}^{3} / N^{+}\left(\Gamma_{2}\right)$ which is regular by [16; 39, Lemma 4]. Thus $N^{+}\left(\Gamma_{2}\right) \subseteq N^{+}\left(\Gamma_{1}\right)$. Similarly $N^{+}\left(\Gamma_{1}\right) \subseteq N^{+}\left(\Gamma_{2}\right)$, so these normalizers are equal. This completes the proof.

An immediate corollary is a strengthened version of [9, Theorem 2.2] for hyperbolic knots in $S^{3}$.

Corollary 4.2 Two hyperbolic knot complements are cyclically commensurable if and only if they have a common regular finite cover with a single cusp.

Proof The forward implication is obvious, as a finite cyclic cover of a knot complement has one cusp. Let $K_{1}$ and $K_{2}$ be two hyperbolic knots in $S^{3}$. Let $N$ be the common covering of their complements, with a single cusp $C$. Let $G_{1}$ and $G_{2}$ be the two associated covering groups. Then the subgroup $G \subset \operatorname{Isom}^{+}(N)$ generated by $G_{1}$ 
and $G_{2}$ is finite and acts identically on $H_{1}(C, \mathcal{Z})$, since $G_{1}$ and $G_{2}$ do. So the quotient orbifold $\mathcal{O}=N / G$ has a single torus cusp. By [16; 39, Lemma 4] the coverings $S^{3} \backslash K_{1} \rightarrow \mathcal{O}$ and $S^{3} \backslash K_{2} \rightarrow \mathcal{O}$ are cyclic. Hence Proposition 4.1 shows that $S^{3} \backslash K_{1}$ and $S^{3} \backslash K_{2}$ are cyclically commensurable.

The following result is a consequence of the fact that a knot complement has a unique 2-fold covering.

Lemma 4.3 Let $K$ be a hyperbolic knot. If $K$ is strongly invertible, $\mathcal{Z}_{K}$ is the unique 2 -fold covering of $\mathcal{O}(K)=\mathbf{H}^{3} / N^{+}(K)$ with a torus cusp, up to an orientationpreserving homeomorphism.

Proof Set $n=|Z(K)|$ and let $D_{n}$ denote the dihedral group of order $2 n$. By hypothesis, $\operatorname{Isom}^{+}\left(S^{3} \backslash K\right) \cong D_{n}$. We have the exact sequence

$$
1 \rightarrow \pi_{1}\left(S^{3} \backslash K\right) \rightarrow \pi_{1}(\mathcal{O}(K)) \stackrel{\varphi}{\rightarrow} D_{n} \rightarrow 1 .
$$

Let $\mathcal{O}^{\prime}$ be a two-fold cover of $\mathcal{O}(K)$ with a torus cusp. Then $\pi_{1}\left(\mathcal{O}^{\prime}\right)$ is an index 2 subgroup of $\pi_{1}(\mathcal{O}(K))$ whose image by $\varphi$ is a subgroup $G$ of $D_{n}$ of index 1 or 2. It is evident that $\operatorname{ker}\left(\varphi \mid \pi_{1}\left(\mathcal{O}^{\prime}\right)\right)=\pi_{1}\left(S^{3} \backslash K\right) \cap \pi_{1}\left(\mathcal{O}^{\prime}\right)$ and $\left[\pi_{1}\left(S^{3} \backslash K\right)\right.$ : $\left.\operatorname{ker}\left(\varphi \mid \pi_{1}\left(\mathcal{O}^{\prime}\right)\right)\right]\left[D_{n}: G\right]=2$.

If $G=D_{n}$, then $\left[\pi_{1}\left(S^{3} \backslash K\right): \operatorname{ker}\left(\varphi \mid \pi_{1}\left(\mathcal{O}^{\prime}\right)\right)\right]=2$, so $\operatorname{ker}\left(\varphi \mid \pi_{1}\left(\mathcal{O}^{\prime}\right)\right)=\pi_{1}(M)$ where $M$ is the unique 2-fold cyclic cover of $S^{3} \backslash K$. There is a regular cover $M \rightarrow \mathcal{O}^{\prime}$ of group $G=D_{n}$. Thus a strong inversion $\sigma \in \operatorname{Isom}^{+}\left(S^{3} \backslash K\right)$ lifts to an involution $\widetilde{\sigma}$ of $M$. Since $M$ has one end, which is a torus cusp, it is easy to see that $\widetilde{\sigma}$ acts on its first homology by multiplication by -1 . But then $\mathcal{O}^{\prime}$ has an $S^{2}(2,2,2,2)$ cusp, contrary to our hypotheses.

Thus $\left[D_{n}: G\right]=2$, so $|G|=|Z(K)|$ and $\operatorname{ker}\left(\varphi \mid \pi_{1}\left(\mathcal{O}^{\prime}\right)\right)=\pi_{1}\left(S^{3} \backslash K\right)$. Therefore $S^{3} \backslash K$ covers $\mathcal{O}^{\prime}$ regularly with group $G \leq D_{n}=\operatorname{Isom}^{+}\left(S^{3} \backslash K\right)$. Since $\mathcal{O}^{\prime}$ has a torus cusp, $G$ acts freely on $K$, and so as $|G|=|Z(K)|, G=Z(K)$. Thus $\mathcal{O}^{\prime}=\left(S^{3} \backslash K\right) / Z(K)=\mathcal{Z}_{K}$, which is what we needed to prove.

Remark 4.4 The method of proof of the previous lemma yields the following stronger result: Let $K$ be a hyperbolic knot and $S^{3} \backslash K \rightarrow \mathcal{O}$ a cover where $\mathcal{O}$ is an orientable 3 -orbifold with an $S^{2}(2,2,2,2)$ cusp. Then there is a unique 2-fold cover $\mathcal{O}^{\prime} \rightarrow \mathcal{O}$ such that $\mathcal{O}^{\prime}$ has a torus cusp.

The following proposition and Proposition 4.1 immediately imply assertion (1) of Theorem 1.4. 
Proposition 4.5 Two hyperbolic knots $K$ and $K^{\prime}$ without hidden symmetries are commensurable if and only if there is an orientation-preserving homeomorphism between $\mathcal{Z}_{K}$ and $\mathcal{Z}_{K^{\prime}}$. In particular $K$ and $K^{\prime}$ are commensurable if and only if they are cyclically commensurable.

Proof If there is an orientation-preserving homeomorphism between $\mathcal{Z}_{K}$ and $\mathcal{Z}_{K^{\prime}}$ then clearly $K$ and $K^{\prime}$ are commensurable, and in fact cyclically commensurable by Proposition 4.1. We prove the converse by distinguishing two cases:

(a) $K$ is not strongly invertible. Then $\operatorname{Isom}^{+}\left(S^{3} \backslash K\right)=Z(K)$ and since $K$ has no hidden symmetries, $\mathcal{O}_{\min }(K)=\left(S^{3} \backslash K\right) / Z(K)=\mathcal{Z}_{K}$. In particular $\mathcal{O}_{\min }(K)$ has a torus cusp. Hence if $K^{\prime}$ is commensurable with $K, K^{\prime}$ is not strongly invertible. It follows that $\mathcal{Z}_{K^{\prime}}$ is orientation-preserving homeomorphic to $\mathcal{O}_{\min }(K)=\mathcal{Z}_{K}$.

(b) $K$ is strongly invertible. In this case $\mathcal{O}_{\min }(K)=\left(S^{3} \backslash K\right) / \operatorname{Isom}^{+}\left(S^{3} \backslash K\right)$ has a flexible cusp with horospherical section $S^{2}(2,2,2,2)$. Hence any knot $K^{\prime}$ commensurable with $K$ is strongly invertible. The result follows from Lemma 4.3 as $\mathcal{Z}_{K}$ and $\mathcal{Z}_{K^{\prime}}$ are 2 -fold coverings of $\mathcal{O}_{\min }(K)$ with torus cusps.

Now suppose that $K$ and $K^{\prime}$ are commensurable knots without hidden symmetries. The proof shows that $S^{3} \backslash K$ and $S^{3} \backslash K^{\prime}$ each cyclically cover $\mathcal{Z}_{K}$. Thus $K$ and $K^{\prime}$ are cyclically commensurable by Proposition 4.1.

The following theorem is a main step in our study. It immediately implies Theorem 1.4. Recall that the meridional slope of $S^{3} \backslash K$ projects to a slope $r(K)$ in the torus cusp of $\mathcal{Z}_{K}=\left(S^{3} \backslash K\right) / Z(K)$.

Theorem 4.6 Suppose that $K$ is a hyperbolic knot and let $K^{\prime}$ be a knot cyclically commensurable with $K$.

(1) There is an orientation-preserving homeomorphism between $\mathcal{Z}_{K}$ and $\mathcal{Z}_{K^{\prime}}$.

(2) If $r(K)$ and $r\left(K^{\prime}\right)$ coincide under some orientation-preserving homeomorphism between $\mathcal{Z}_{K}$ and $\mathcal{Z}_{K^{\prime}}$, then $K$ and $K^{\prime}$ are equivalent knots.

(3) If $f_{K^{\prime}}: \mathcal{Z}_{K^{\prime}} \rightarrow \mathcal{Z}_{K}$ is a homeomorphism and $r_{K^{\prime}}$ is the slope in the cusp of $\mathcal{Z}_{K}$ determined by $f_{K^{\prime}}\left(r\left(K^{\prime}\right)\right)$, then $\Delta\left(r(K), r_{K^{\prime}}\right) \leq 1$.

Proof of Theorem 1.4 By Assertion (1) of Theorem 4.6 we can fix an orientationpreserving homeomorphism $f_{K^{\prime}}: \mathcal{Z}_{K^{\prime}} \rightarrow \mathcal{Z}_{K}$ for each $K^{\prime} \in \mathcal{C C}(K)$. Let $r_{K^{\prime}}$ be the slope in the cusp of $\mathcal{Z}_{K}$ determined by $f_{K^{\prime}}\left(r\left(K^{\prime}\right)\right)$. Assertion (3) implies that there are at most three slopes in the set $\left\{r_{K^{\prime}}: K^{\prime} \in \mathcal{C C}(K)\right\}$, while assertion (2) implies that the function which associates the slope $r_{K^{\prime}}$ to $K^{\prime} \in \mathcal{C}(K)$ is injective. Thus Theorem 1.4 holds. 
Assertion (1) of Theorem 4.6 is the content of the following proposition:

Proposition 4.7 Two hyperbolic knots $K$ and $K^{\prime}$ are cyclically commensurable if and only if there is an orientation-preserving homeomorphism between $\mathcal{Z}_{K}$ and $\mathcal{Z}_{K^{\prime}}$.

Proof By Proposition 4.1 if the hyperbolic knots $K$ and $K^{\prime}$ are cyclically commensurable then there is an orientation-preserving homeomorphism between the orbifolds $\mathcal{O}(K)=\mathbf{H}^{3} / N^{+}(K)$ and $\mathcal{O}\left(K^{\prime}\right)=\mathbf{H}^{3} / N^{+}\left(K^{\prime}\right)$. Then the proof is the same as the proof of Proposition 4.5 by considering $\mathcal{O}(K)$ instead of $\mathcal{O}_{\min }(K)$.

Assertion (2) of Theorem 4.6 is given by the following lemma:

Lemma 4.8 Let $K$ and $K^{\prime}$ be two hyperbolic cyclically commensurable knots. If $r(K)$ and $r\left(K^{\prime}\right)$ coincide under some orientation-preserving homeomorphism between $\mathcal{Z}_{K}$ and $\mathcal{Z}_{K^{\prime}}$, then $K$ and $K^{\prime}$ are equivalent knots.

Proof Suppose $r(K)$ and $r\left(K^{\prime}\right)$ coincide under some homeomorphism $\mathcal{Z}_{K} \rightarrow \mathcal{Z}_{K^{\prime}}$. Then we have an induced homeomorphism $f:\left(\mathcal{Z}_{K}(r(K)), \mathcal{Z}_{K}\right) \rightarrow\left(\mathcal{Z}_{K^{\prime}}\left(r\left(K^{\prime}\right)\right), \mathcal{Z}_{K^{\prime}}\right)$. By construction, $\mathcal{Z}_{K}(r(K)) \cong S^{3} / Z(K)$ so

$$
\pi: S^{3} \rightarrow S^{3} / Z(K)=\mathcal{Z}_{K}(r(K))
$$

is a universal cover. In the same way

$$
\pi^{\prime}: S^{3} \rightarrow S^{3} / Z\left(K^{\prime}\right)=\mathcal{Z}_{K^{\prime}}\left(r\left(K^{\prime}\right)\right)
$$

is a universal cover. Since universal covers are unique up to covering equivalence, there is a homeomorphism (preserving orientation) $\tilde{f}: S^{3} \rightarrow S^{3}$ such that $\pi^{\prime} \circ \tilde{f}=f \circ \pi$. In particular,

$$
\left.\tilde{f}\left(S^{3} \backslash K\right)=\tilde{f}\left(\pi^{-1}\left(\mathcal{Z}_{K}\right)\right)=\pi^{\prime-1}\left(f\left(\mathcal{Z}_{K}\right)\right)\right)=\pi^{\prime-1}\left(\mathcal{Z}_{K^{\prime}}\right)=S^{3} \backslash K^{\prime} .
$$

Thus the complement of $K$ is orientation-preserving homeomorphic to the complement of $K^{\prime}$, so $K$ is equivalent to $K^{\prime}[19]$.

With the notation of Lemma 4.8, Assertion (3) of Theorem 4.6 is the content of the following lemma:

Lemma 4.9 Let $K$ and $K^{\prime}$ be two cyclically commensurable knots and $f: \mathcal{Z}_{K^{\prime}} \rightarrow \mathcal{Z}_{K}$ a homeomorphism ${ }^{1}$. Then $\Delta\left(r(K), r_{K^{\prime}}\right) \leq 1$ where $r_{K^{\prime}}$ is the slope in the cusp of $\mathcal{Z}_{K}$ corresponding to $f\left(r\left(K^{\prime}\right)\right)$.

\footnotetext{
${ }^{1}$ We do not assume that $f$ preserves orientation.
} 


\section{Proof Set}

$$
\mathcal{Z}_{K}^{0}=\mathcal{Z}_{K} \backslash N\left(\Sigma\left(\mathcal{Z}_{K}\right)\right)
$$

where $N\left(\Sigma\left(\mathcal{Z}_{K}\right)\right)$ denotes a small, open tubular neighborhood of $\Sigma\left(\mathcal{Z}_{K}\right)$. Then $\mathcal{Z}_{K}^{0}$ has no singularities. Since $\Sigma\left(\mathcal{Z}_{K}\right)$ is a geodesic link in the hyperbolic orbifold $\mathcal{Z}_{K}$, $\mathcal{Z}_{K}^{0}$ admits a complete, finite volume, hyperbolic structure [41;24].

By the geometrization of finite group actions [5; 27], we can suppose that $Z(K)$ and $Z\left(K^{\prime}\right)$ act orthogonally on $S^{3}$. It follows that both Dehn fillings of the torus cusp of $\mathcal{Z}_{K}$ along the slopes $r(K)$ and $r_{K^{\prime}}$ give orbi-lens spaces $\mathcal{L}_{K}=\mathcal{Z}_{K}(r(K))=$ $S^{3} / Z(K)$ and $\mathcal{L}^{\prime}=\mathcal{Z}_{K}\left(r_{K^{\prime}}\right) \cong \mathcal{Z}_{K^{\prime}}\left(r\left(K^{\prime}\right)\right)=S^{3} / Z\left(K^{\prime}\right)$. By Corollary 3.2, $\left|\mathcal{L}_{K}\right|$ and $\left|\mathcal{L}^{\prime}\right|$ are lens spaces, possibly $S^{3}$. Moreover the singular set $\Sigma\left(\mathcal{L}_{K}\right)$, resp. $\Sigma\left(\mathcal{L}^{\prime}\right)$, is either empty or a sublink of the union of the cores of the two solid tori in a genus 1 Heegaard splitting of $\left|\mathcal{L}_{K}\right|$, resp. $\left|\mathcal{L}^{\prime}\right|$. Since $\mathcal{Z}_{K}^{0}(r(K))=\mathcal{L}_{K} \backslash N\left(\Sigma\left(\mathcal{Z}_{K}\right)\right)=$ $\mathcal{L}_{K} \backslash N\left(\Sigma\left(\mathcal{L}_{K}\right)\right)$, we have

$$
\mathcal{Z}_{K}^{0}(r(K)) \cong \begin{cases}\left|\mathcal{L}_{K}\right| & \text { if }\left|\Sigma\left(\mathcal{Z}_{K}\right)\right|=0 \\ S^{1} \times D^{2} & \text { if }\left|\Sigma\left(\mathcal{Z}_{K}\right)\right|=1 \\ S^{1} \times S^{1} \times[0,1] & \text { if }\left|\Sigma\left(\mathcal{Z}_{K}\right)\right|=2\end{cases}
$$

In the same way,

$$
\mathcal{Z}_{K}^{0}\left(r_{K^{\prime}}\right) \cong \begin{cases}\left|\mathcal{L}^{\prime}\right| & \text { if }\left|\Sigma\left(\mathcal{Z}_{K}\right)\right|=0 \\ S^{1} \times D^{2} & \text { if }\left|\Sigma\left(\mathcal{Z}_{K}\right)\right|=1 \\ S^{1} \times S^{1} \times[0,1] & \text { if }\left|\Sigma\left(\mathcal{Z}_{K}\right)\right|=2\end{cases}
$$

One can choose slopes on the components $\partial N(\Sigma(\mathcal{Z})) \subset \partial \mathcal{Z}_{K}^{0}$ such that $M$, the manifold obtained by Dehn filling $\mathcal{Z}_{K}^{0}$ along these slopes, is hyperbolic. It follows from above that $M(r(K))$ and $M\left(r^{\prime}\right)$ have cyclic fundamental groups, so the cyclic surgery theorem [10] implies that $\Delta\left(r(K), r_{K^{\prime}}\right) \leq 1$.

This completes the proof of Theorem 4.6, and therefore of Theorem 1.4.

We have the following consequence of the proof. A good orbifold is an orbifold which is covered by a manifold.

Scholium 4.10 Let $M$ be a hyperbolic orbifold with a single torus cusp. If $M\left(r_{1}\right)$ and $M\left(r_{2}\right)$ yield good orbifolds with cyclic orbifold fundamental group, then $\Delta\left(r_{1}, r_{2}\right) \leq 1$. In particular, there are at most 3 such slopes.

Proof Suppose that the group $\pi_{1}^{\text {orb }}\left(M\left(r_{1}\right)\right)$ is finite cyclic. Then the universal cover is $S^{3}$ and $M$ is the complement of a knot in an orbi-lens space, and the result follows from the proof of Lemma 4.9 . 
Suppose $\pi_{1}^{\text {orb }}\left(M\left(r_{1}\right)\right)$ is infinite cyclic. Since its universal cover is a manifold and its fundamental group has no torsion, $M\left(r_{1}\right)$ is a manifold and hence $M$ is a hyperbolic manifold. The result follows from the Cyclic Surgery Theorem [10].

The analysis of the action on the knot complement by a cyclic group of symmetries as in Lemma 4.9 above along with an observation of M Kapovich yield the following characterisation of the minimal element in the commensurability class of a knot complement.

Corollary 4.11 If $\mathcal{O}_{\min }(K)$ is the minimal element of a nonarithmetic commensurability class which contains a knot complement $S^{3} \backslash K$ then the underlying space of $\mathcal{O}_{\min }(K)$ is either an open ball or the complement of a knot in a lens space.

Proof Let $\mathcal{O}_{\min }(K)$ be the minimal element of the commensurability class and $\widehat{\mathcal{O}}_{\text {min }}(K)$ the associated orbifold with boundary obtained by truncating along the cusp. Since the boundary of $S^{3} \backslash N(K)$ is a torus, $\partial \hat{\mathcal{O}}_{\min }(K)$ is a closed orientable Euclidean 2-orbifold, which implies that it either a torus or has underlying space $S^{2}$. When $\partial \widehat{\mathcal{O}}_{\min }(K)$ is a torus, the covering is a regular cyclic covering by $[16 ; 39$, Lemma 4]. Therefore, our analysis in Lemma 4.9 implies that the underlying space of $\widehat{\mathcal{O}}_{\min }(K)$ is a lens space with a regular neighborhood of a knot removed, and that $\left|\mathcal{O}_{\min }(K)\right|$ is the complement of a knot in a lens space. The case when $\left|\partial \widehat{\mathcal{O}}_{\min }(K)\right|$ is $S^{2}$ is an observation of M Kapovich. There is a map which is the composition $S^{3} \backslash N(K) \rightarrow \widehat{\mathcal{O}}_{\text {min }}(K) \rightarrow\left|\widehat{\mathcal{O}}_{\text {min }}(K)\right|$. The image of $\pi_{1}\left(S^{3} \backslash N(K)\right)$ under the induced homomorphism is trivial, as $\pi_{1}\left(S^{3} \backslash N(K)\right)$ is normally generated by a meridian. Therefore if $\left|\widehat{\mathcal{O}}_{\min }(K)\right|$ has any nontrivial cover (such as the universal cover) the above map $S^{3} \backslash N(K) \rightarrow\left|\widehat{\mathcal{O}}_{\text {min }}(K)\right|$ lifts to this cover, which is a contradiction as any nontrivial cover of a manifold with boundary $S^{2}$ has multiple boundary components. Therefore $\left|\widehat{\mathcal{O}}_{\min }(K)\right|$ has trivial fundamental group and by work of Perelman (see Morgan and Tian [27]) it is a ball. So $\mathcal{O}_{\min }(K)$ has underlying space an open ball.

By [3, Main Theorem] (see also [28]), $\Gamma \in \operatorname{Isom}^{+}\left(\mathbf{H}^{3}\right)$ is generated by rotations exactly when the underlying space of $\mathbf{H}^{3} / \Gamma$ is simply connected. Therefore we have the following corollary of Corollary 4.11.

Corollary 4.12 A noninvertible hyperbolic knot $K$ has a hidden symmetry if and only if its group $\pi_{1}\left(S^{3} \backslash K\right)$ is commensurable with a Kleinian group generated by rotations.

The following proposition is a consequence of the proof of Theorem 4.6. It states that a hyperbolic knot $K$ is not unique in its cyclic commensurability class if and only if $\bar{K} \subset \mathcal{L}_{K}$ admits a nontrivial orbi-lens space surgery. More precisely: 
Proposition 4.13 A commensurability class contains cyclically commensurable knot complements $S^{3} \backslash K$ and $S^{3} \backslash K^{\prime}$ where $K^{\prime} \neq K$ if and only if it contains the complement of a knot $\bar{K}$ in an orbi-lens space $\mathcal{L}$ such that $\bar{K}$ is primitive in $\mathcal{L}$ and $\mathcal{L}$ admits a nontrivial orbi-lens space surgery $\mathcal{L}^{\prime}$ along $\bar{K}$. We may take $\mathcal{L} \backslash \bar{K}$ to be $\mathcal{Z}_{k}$, with slopes $r(K)$ and $r^{\prime}$ yielding the lens spaces $\mathcal{L}$ and $\mathcal{L}^{\prime}$ respectively. If $\pi^{\prime}: S^{3} \rightarrow \mathcal{L}^{\prime}$ is the universal covering and $\bar{K}^{\prime} \subset \mathcal{L}^{\prime}$ is the core of the $r^{\prime}$-Dehn filling of $\mathcal{Z}_{K}$, then $K^{\prime}=\pi^{-1}\left(\bar{K}^{\prime}\right)$.

This result gives a way of constructing every knot cyclically commensurable with $K$. Since the only nonarithmetic knots known to admit hidden symmetries are the two commensurable dodecahedral knots of Aitchison and Rubinstein [1], all the other pairs of commensurable hyperbolic knots constructed so far can be obtained from the construction given in Proposition 4.13.

Proof of Proposition 4.13 We continue to use the notation developed in the proof of Theorem 4.6. Suppose a commensurability class $\mathcal{C}$ contains cyclically commensurable knot complements $S^{3} \backslash K$ and $S^{3} \backslash K^{\prime}$. By the proof of Proposition 4.1 the quotients $\mathcal{Z}_{k}$ and $\mathcal{Z}_{K^{\prime}}$ are homeomorphic. By the proof of Theorem 4.6, there are distinct slopes $r(K)$ and $r_{K^{\prime}}$, of $\mathcal{Z}_{k}$ such that filling along these slopes produces lens spaces $\mathcal{L}_{K}$ and $\mathcal{L}_{K^{\prime}}$ respectively. Also, the preimages of the surgery core $\bar{K}$ in the universal covers of $\mathcal{L}_{K}$ and $\mathcal{L}_{K^{\prime}}$ are the knots $K \subset S^{3}$ and $K^{\prime} \subset S^{3}$. Since $K$ is a knot, $\bar{K}$ is primitive in $\mathcal{L}_{K}$. Thus $\mathcal{Z}_{K}$ satisfies the conclusions of the theorem.

Suppose that a commensurability class $\mathcal{C}$ contains the complement of a knot in an orbilens space $\mathcal{L} \backslash \bar{K}$ where $\bar{K}$ is primitive in $\mathcal{L}$ and $\bar{K}$ admits a nontrivial orbi-lens space surgery. Then by primitivity, the preimage of $\bar{K}$ in the universal cover $S^{3}$ of $\mathcal{L}$ is a knot $K$. Since the covering group $S^{3} \rightarrow \mathcal{L}$ is cyclic, $S^{3} \backslash K$ cyclically covers $\mathcal{L} \backslash \bar{K} \cong \mathcal{O}$. Let $r_{K}$ be the projection of the meridional slope of $S^{3} \backslash K$. Denote the nontrivial orbilens space filling of $\mathcal{L} \backslash \bar{K}$ by $\mathcal{L}^{\prime}$ and the filling slope by $r_{K^{\prime}}$. By the proof of Lemma 4.9, $\Delta\left(r_{K}, r_{K^{\prime}}\right) \leq 1$. Thus a representative curve for $r_{K^{\prime}}$ is isotopic to $\bar{K}$ in $\mathcal{L}$. It follows that representative curves for $r_{K}$ and $r_{K^{\prime}}$ carry the first homology of $\mathcal{L} \backslash \bar{K}$. Thus the core $\bar{K}^{\prime}$ of the $r_{K^{\prime}}$-Dehn filling solid torus in $\mathcal{L}^{\prime}$ carries a generator of $H_{1}\left(\mathcal{L}^{\prime}\right)$ and therefore the preimage of $\bar{K}^{\prime}$ in the universal cover of $\mathcal{L}^{\prime}$ is a knot in $S^{3}$. Furthermore, $S^{3} \backslash K^{\prime}$ cyclically covers $\mathcal{O} \cong \mathcal{L}^{\prime} \backslash \bar{K}^{\prime} \cong \mathcal{L} \backslash \bar{K}$. Therefore, $\mathcal{C}$ contains the cyclically commensurable knots $S^{3} \backslash K$ and $S^{3} \backslash K^{\prime}$. Suppose that $K$ is equivalent to $K^{\prime}$. An orientation-preserving homeomorphism $S^{3} \backslash K \rightarrow S^{3} \backslash K^{\prime}$ induces an orientationpreserving homeomorphism $f: \mathcal{L} \backslash \bar{K} \rightarrow \mathcal{L}^{\prime} \backslash \bar{K}^{\prime}$. It is evident that $f(r(K))=r\left(K^{\prime}\right)$. By construction we have an orientation-preserving homeomorphism $g: \mathcal{L} \backslash \bar{K} \rightarrow \mathcal{L}^{\prime} \backslash \bar{K}^{\prime}$ such that $g\left(r^{\prime}\right)=r\left(K^{\prime}\right)$. Thus $h=g^{-1} \circ f: \mathcal{L} \backslash \bar{K} \rightarrow \mathcal{L} \backslash \bar{K}$ is an orientation-preserving 
homeomorphism such that $h(r(K))=r^{\prime}$. But this is impossible as Lemma 2.1 would then imply that $r^{\prime}=r(K)$. Thus $K$ and $K^{\prime}$ are distinct knots by Theorem 4.6.

This suggests the following conjecture:

Conjecture 4.14 A rigid commensurability class does not contain cyclically commensurable hyperbolic knot complements.

Theorem 1.8 and Proposition 4.13 reduce the characterisation of hyperbolic knots $K \subset S^{3}$ such that $|\mathcal{C C}(K)| \geq 2$ to the case where $Z(K)$ acts freely on $S^{3}$ and to the construction of all primitive knots in a lens space with a nontrivial lens space surgery. We remark that the situation is completely understood for the case of orbi-lens spaces:

Proposition 4.15 Let $\bar{K}$ be a primitive hyperbolic knot in an orbi-lens space $L$ with nontrivial ramification locus $\Sigma(L)$. If a nontrivial Dehn surgery along $\bar{K}$ produces an orbi-lens space, then $K$ is a Berge-Gabai knot in $L \backslash N(\Sigma(L))$.

Proof The fact that the singular locus must have one component is proven below in Proposition 5.1. Let $V_{1} \cup V_{2}$ be the Heegaard splitting of $L$ where $V_{1}$ is a regular neighborhood of $\Sigma(L)$ and $\bar{K} \subset V_{2}$. Assume nontrivial surgery along $\bar{K}$ in $L$ yields an orbi-lens space $L^{\prime}$. By removing neighborhoods of the ramification loci in $L$ and $L^{\prime}$, we see that nontrivial surgery along $\bar{K}$ in $V_{2}$ yields a solid torus. Then by Definition $5.4 \bar{K}$ is a Berge-Gabai knot in $V_{2}=L \backslash N(\Sigma(L))$.

\section{Unwrapped 1-bridge braids}

In this section we prove Theorem 1.8 which characterizes all periodic hyperbolic knots such that $|\mathcal{C C}(K)| \geq 2$.

Recall that a 1-bridge braid in a solid torus $V$ is a braid in $V$ which is 1-bridge with respect to some boundary-parallel torus in $\operatorname{int}(V)$. Connected 1-bridge braids were classified by Gabai [14].

A cosmetic surgery slope of a knot in a 3-manifold $W$ is a slope on the boundary of the exterior of the knot whose associated surgery yields a manifold homeomorphic to $W$. We say that $K$ has a nontrivial cosmetic surgery if it has such a slope which is distinct from the knot's meridian. The following proposition is a consequence of work of Gabai and Gordon-Luecke.

Proposition 5.1 If a hyperbolic knot $K$ in $V \cong S^{1} \times D^{2}$ or $V \cong S^{1} \times S^{1} \times I$ admits a nontrivial cosmetic surgery, then $V \cong S^{1} \times D^{2}$ and $K$ is a 1 -bridge braid. 
Proof First assume that $K$ is a hyperbolic knot in $V \cong S^{1} \times D^{2}$. Gabai [13] has shown that any knot in a solid torus which admits a nontrivial cosmetic surgery is either contained in a 3-ball or is a 0 -bridge braid or is a 1-bridge braid. In our case, hyperbolicity rules out the first two cases. Thus $K$ is a 1 -bridge braid.

The case where $K$ is a hyperbolic knot in $V \cong S^{1} \times S^{1} \times I$ is ruled out by the following lemma:

Lemma 5.2 A hyperbolic knot $K$ in $V \cong S^{1} \times S^{1} \times I$ admits no nontrivial cosmetic surgery.

Proof Assume that there is a nontrivial cosmetic surgery $r$ for $K$. Then $r$ is a nontrivial cosmetic surgery slope when $K$ is considered as a knot in any Dehn filling of $V$ along $T^{2} \times 0$. Choose such a filling in which $K$ remains hyperbolic. The previous argument then implies that $K$ is not homologically trivial in the Dehn filling of $V$, and therefore not in $V$ as well. Then there is an essential simple closed curve $C \subset T^{2} \times 0$ such that the class in $H_{1}(V)$ carried by $K$ is an integral multiple of that carried by $C$. Since the algebraic intersection of $K$ with the properly embedded, essential annulus $A=C \times I \subset V$ is null, $A$ defines a homology class $[A] \in H_{2}(V \backslash K, \partial V) \cong \mathbb{Z}$. Let $(F, \partial F) \subset(V \backslash K, \partial V)$ be a norm minimizing surface representing the homology class $[A]$. By a result of Gabai [12, Corollary], $F$ remains norm minimizing in all manifolds obtained by Dehn surgeries along $K$ except at most one. Since two such surgeries yield manifolds homeomorphic to $S^{1} \times S^{1} \times I, F$ must be an essential annulus, contrary to the hypothesis that $K$ is hyperbolic in $V$. Thus the lemma holds.

This completes the proof of Proposition 5.1.

Recall the hyperbolic manifold

$$
\mathcal{Z}_{K}^{0}=\mathcal{Z}_{K} \backslash N\left(\Sigma\left(\mathcal{Z}_{K}\right)\right)
$$

defined in the proof of Lemma 4.9. It follows from this proof that if $|\mathcal{C C}(K)|>1$ and $\left|\Sigma\left(\mathcal{Z}_{K}\right)\right| \geq 1$, then the core $\bar{K}$ of the Dehn filling $\mathcal{Z}_{K}^{0}(r(K)) \cong S^{1} \times D^{2}$ or $S^{1} \times S^{1} \times I$ admits a nontrivial cosmetic surgery. Hence Proposition 5.1 immediately implies the following corollary:

Corollary 5.3 If $K$ is a periodic hyperbolic knot and $\left|\Sigma\left(\mathcal{Z}_{K}\right)\right|=2$, then $|\mathcal{C C}(K)|=1$. In particular, if $K$ has no hidden symmetry $|\mathcal{C}(K)|=1$.

This result implies assertion (1) of Theorem 1.8. Next we examine the case $\left|\Sigma\left(\mathcal{Z}_{K}\right)\right|=1$. 
Definition 5.4 A Berge-Gabai knot in a solid torus is a 1-bridge braid in a solid torus which admits a nontrivial cosmetic surgery slope.

The winding number of a Berge-Gabai knot in a solid torus is the braid index of its associated 1-bridge braid.

Berge-Gabai knots and their cosmetic surgery slopes have been classified. See [4; 14]. Moreover, it follows from the description given in [14] that these knots can be embedded in $S^{3}$ as homogeneous braids and hence as fibred knots by Stallings [43].

Definition 5.5 (1) Let $w, p, q, a$ be integers with $w, a, p \geq 1$ and $\operatorname{gcd}(p, q)=$ $\operatorname{gcd}(w, a p)=1$. A Berge-Gabai knot $\bar{K}$ of winding number $w$ in $\mathcal{L}(p, q ; a)$ consists of a knot $\bar{K} \subset \mathcal{L}(p, q ; a)$ and a genus one Heegaard splitting $V_{1} \cup V_{2}$ of $|\mathcal{L}(p, q ; a)|$ such that $\bar{K}$ is a Berge-Gabai knot of winding number $w$ in $V_{1}$ and $\Sigma(\mathcal{L}(p, q ; a))$ is a closed submanifold of the core of $V_{2}$.

(2) A $(p, q ; a)$-unwrapped Berge-Gabai knot in $S^{3}$ is a knot in $S^{3}$ which is the inverse image of a Berge-Gabai knot in $\mathcal{L}(p, q ; a)$ under the universal cover $S^{3} \rightarrow \mathcal{L}(p, q ; a)$.

Note that the inverse image in $S^{3}$ of a Berge-Gabai knot in $\mathcal{L}(p, q ; a)$ is a knot (ie connected) as its winding number $w$ is coprime to ap .

Lemma 5.6 Suppose that $\bar{K}$ is a Berge-Gabai knot of winding number $w$ in $\mathcal{L}(p, q ; a)$ where $p \geq 1$ is coprime with $w$. Let $V_{1} \cup V_{2}$ be a Heegaard splitting of $L(p, q)$ where $\bar{K} \subset \operatorname{int}\left(V_{1}\right)$ and $\Sigma(\mathcal{L}(p, q ; a))$ is a closed submanifold of the core of $V_{2}$. If $r$ is a nontrivial cosmetic surgery slope of $\bar{K}$ considered as a knot in $V_{1}$, then $\bar{K}(r) \cong \mathcal{L}\left(p^{\prime}, q^{\prime} ; a\right)$ where $\operatorname{gcd}\left(p, p^{\prime}\right)=1$.

Proof It is clear that $|\bar{K}(r)|$ has Heegaard genus one, so is $L\left(p^{\prime}, q^{\prime}\right)$ for some $p^{\prime} \geq 0$. (We take the convention that $L\left(0, q^{\prime}\right) \cong S^{1} \times S^{2}$.) We must show $p^{\prime}$ is nonzero and relatively prime to $p$.

Let $W$ be the exterior of $\bar{K}$ in $V_{1}$ and write $\partial W=T_{0} \cup T_{1}$ where $T_{1}=\partial V_{1}$ and $T_{0}$ is the boundary of a tubular neighborhood of $\bar{K}$. There are bases $\mu_{0}, \lambda_{0}$ of $H_{1}\left(T_{0}\right)$ and $\mu_{1}, \lambda_{1}$ of $H_{1}\left(T_{1}\right)$ such that $\mu_{0}$ is a meridian of $\bar{K}, \mu_{1}$ is a meridian of $V_{1}$, and $\mu_{1}=w \mu_{0}, \lambda_{0}=w \lambda_{1}$ in $H_{1}(W)$.

It is shown in [14, Lemma 3.2] that $r= \pm\left(m \mu_{0}+\lambda_{0}\right)$ where $\operatorname{gcd}(m, w)=1$. A homological calculation (see [18, Lemma 3.3]) shows that $\mu_{1}(r)$, the meridian slope 
of the solid torus $\left(V_{1}, \bar{K}\right)(r)$, is given by $\mu_{1}(r)=m \mu_{1}+w^{2} \lambda_{1}$. By hypothesis, $q \mu_{1}+p \lambda_{1}$ is the meridian of $V_{2}$ and therefore

$$
p^{\prime}=\Delta\left(\mu_{1}(r), q \mu_{1}+p \lambda_{1}\right)=\Delta\left(m \mu_{1}+w^{2} \lambda_{1}, q \mu_{1}+p \lambda_{1}\right)=\left|m p-q w^{2}\right| .
$$

Since $p$ is coprime to $q$ and $w^{2}$, it is coprime to $\left|m p-q w^{2}\right|$, and since $p \geq 1$ and $\operatorname{gcd}(m, w)=1,\left|m p-q w^{2}\right| \neq 0$. Thus the lemma holds.

Next we characterize periodic hyperbolic knots $K$ such that $\left|\Sigma\left(\mathcal{Z}_{K}\right)\right|=1$ and $|\mathcal{C C}(K)| \geq 2$. This will finish the proof of Theorem 1.8.

Proposition 5.7 Let $K$ be a hyperbolic knot in $S^{3}$.

(1) If $K$ is periodic such that $\left|\Sigma\left(\mathcal{Z}_{K}\right)\right|=1$ and $|\mathcal{C C}(K)| \geq 2$ then:

(a) $S^{3} / Z(K)=\mathcal{L}(p, q ; a)$, where $a p=|Z(K)|$ and the image $\bar{K}$ of $K$ in $\mathcal{L}(p, q ; a)$ is a Berge-Gabai knot of winding number prime to $|Z(K)|$. Thus $K$ is the $(p, q ; a)$-unwrapped Berge-Gabai knot associated to the core of the surgery torus in $\mathcal{Z}_{K}^{0}(r(K)) \cong S^{1} \times D^{2}$.

(b) $K$ is strongly invertible.

(c) each $K^{\prime} \in \mathcal{C C}(K) \backslash K$ is a $\left(p^{\prime}, q^{\prime} ; a\right)$-unwrapped Berge-Gabai knot associated to the core of the surgery solid torus in $\mathcal{Z}_{K}^{0}\left(r^{\prime}\right)$, where $\left|Z\left(K^{\prime}\right)\right|=a p^{\prime}$, $\operatorname{gcd}\left(p, p^{\prime}\right)=1$, and $r^{\prime}=f\left(r\left(K^{\prime}\right)\right)$ where $f: \mathcal{Z}_{K^{\prime}} \rightarrow \mathcal{Z}_{K}$ is an orientationpreserving homeomorphism.

(2) If $K$ is a $(p, q ; a)$-unwrapped Berge-Gabai knot, then $|\mathcal{C C}(K)| \geq 2$.

This result holds for a periodic hyperbolic knot $K$ without hidden symmetries and any $K^{\prime} \in \mathcal{C}(K) \backslash K$.

Proof First suppose that $K$ is a knot without hidden symmetries such that $\left|\Sigma\left(\mathcal{Z}_{K}\right)\right|=1$ and $|\mathcal{C C}(K)|>1$. Corollary 3.2 shows that $S^{3} / Z(K)$ is an orbi-lens space $\mathcal{L}(p, q ; a)$ where $a p=|Z(K)|$. Let $\bar{K}$ be the image of $K$ in $\mathcal{L}(p, q ; a)$. There is a genus one Heegaard splitting $V_{1} \cup V_{2}$ of $L(p, q)$ such that $V_{1}=\mathcal{Z}_{K}^{0}(r(K))$ and $V_{2}$ is a regular neighborhood of $\Sigma(\mathcal{L}(p, q ; a))=\Sigma\left(\mathcal{Z}_{K}\right)$. It follows from Theorem 4.6 and the proof of Lemma 4.9 that for $K \neq K^{\prime} \in \mathcal{C}(K)$, the image of $r\left(K^{\prime}\right)$ in the cusp of $\mathcal{Z}_{K}$ under a homeomorphism $\mathcal{Z}_{K^{\prime}} \rightarrow \mathcal{Z}_{K}$ is a nontrivial cosmetic surgery slope of $\bar{K}$ in $V_{1}$. Hence, $\bar{K}$ is a Gabai-Berge knot in $V_{1}$, and as its inverse image in $S^{3}$ is $K$, it has winding number coprime to $a p=|Z(K)|$. Thus $K$ is a $(p, q ; a)$-unwrapped Berge-Gabai knot.

As $\bar{K}$ is 1 -bridge braid in $V_{1}$, it lies on a genus 2 Heegaard surface of $L(p, q)$ (cf the proof of Theorem 6.1). Thus $\mathcal{L}(p, q ; a)$ admits an orientation-preserving involution 
which reverses the orientation of $\bar{K}$. Hence $\mathcal{Z}_{K}=\left(S^{3} \backslash K\right) / Z(K)$ is not minimal in its commensurability class. So $\operatorname{Isom}^{+}\left(S^{3} \backslash K\right) \neq Z(K)$, so $K$ is strongly invertible.

Consider $K^{\prime} \in \mathcal{C}(K) \backslash K$. Since the hypotheses hold for $K^{\prime}$ in place of $K$, we see that $K^{\prime}$ is the $\left(p^{\prime}, q^{\prime} ; a\right)$-unwrapped Berge-Gabai knot associated to the core $\bar{K}^{\prime}$ of the surgery solid torus in $\mathcal{Z}_{K}^{0}\left(r^{\prime}\right)$ where $\left|Z\left(K^{\prime}\right)\right|=a p^{\prime}$ and $r^{\prime}$ is the image in the cusp of $\mathcal{Z}_{K}$ of $r\left(K^{\prime}\right)$ under an orientation-preserving homeomorphism $f: \mathcal{Z}_{K^{\prime}} \rightarrow \mathcal{Z}_{K}$ (cf Proposition 4.5). Lemma 5.6 implies that $\operatorname{gcd}\left(p, p^{\prime}\right)=1$. This completes the proof of assertion (1).

Next we prove assertion (2). Suppose that $K$ is a $(p, q ; a)$-unwrapped Berge-Gabai knot. If $a p=1$, then $\mathcal{L}(p, q ; a)=S^{3}$. Lemma 5.6 and [25] show that $K$ has a slope $r$ such that $K(r)$ is a lens space whose fundamental group is nontrivial. This case of assertion (2) then follows from Proposition 4.13.

If $a p>1$, there is a Berge-Gabai knot $\bar{K}$ in $\mathcal{L}(p, q ; a)$ whose inverse image under the universal cover $S^{3} \rightarrow \mathcal{L}(p, q ; a)$ is $K$. Since Berge-Gabai knots in solid tori admit nontrivial cosmetic surgeries, Lemma 5.6 implies that there is a nontrivial slope $r$ of $\bar{K}$ such that $\bar{K}(r) \cong \mathcal{L}\left(p^{\prime}, q^{\prime} ; a\right)$ where $\operatorname{gcd}\left(p, p^{\prime}\right)=1$. This final case of assertion (2) now follows from Proposition 4.13.

We conclude this section with the observation that the characterisation in Proposition 5.7 allows us to show that hyperbolic knot complements with the same volume are not cyclically commensurable.

Proposition 5.8 Let $K$ be a hyperbolic knot with $|\mathcal{C C}(K)| \geq 2$. Then:

(1) the volume of $K$ is different from that of any $K^{\prime} \in \mathcal{C C}(K) \backslash K$.

(2) the only mutant of $K$ contained in $\mathcal{C C}(K)$ is $K$.

(3) if $K$ is commensurable with its mirror image, it is amphichiral.

This result holds for a hyperbolic knot $K$ without hidden symmetries and any $K^{\prime} \in$ $\mathcal{C}(K) \backslash K$.

Proof First we prove that if $K^{\prime} \in \mathcal{C C}(K)$ is distinct from $K$, then the cyclic groups $Z(K)$ and $Z\left(K^{\prime}\right)$ have distinct orders. This will imply that $K$ and $K^{\prime}$ have distinct volumes since $\operatorname{vol}\left(S^{3} \backslash K\right)=|Z(K)| \operatorname{vol}\left(\mathcal{Z}_{K}\right) \neq\left|Z\left(K^{\prime}\right)\right| \operatorname{vol}\left(\mathcal{Z}_{K}\right)=\operatorname{vol}\left(S^{3} \backslash K^{\prime}\right)$.

Suppose that $Z(K)$ acts freely on $S^{3}$. Then $\mathcal{L}_{K}$ is a lens space of the form $L(c, d)$ where $c=|Z(K)|$. Let $M$ denote the exterior of $\bar{K}$ in $L(c, d)$ and note that as $\bar{K}$ is primitive, $H_{1}(M) \cong \mathbb{Z}$. Hence there is a basis $\bar{\mu}, \bar{\lambda}$ of $H_{1}(\partial M)$ such that the image 
of $\bar{\mu}$ in $H_{1}(M)$ generates while the image of $\bar{\lambda}$ is trivial. Clearly, the meridional slope of $\bar{K}$ represents $c \bar{\mu}+e \bar{\lambda}$ in $H_{1}(\partial M)$ for some integer $e$. Similarly $\mathcal{L}_{K^{\prime}}$ is a lens space $L\left(c^{\prime}, d^{\prime}\right)$ where $c^{\prime}=\left|Z\left(K^{\prime}\right)\right|$, so the meridional slope of $\bar{K}^{\prime}$ represents $c^{\prime} \bar{\mu}+e^{\prime} \bar{\lambda}$. The cyclic surgery theorem [10] implies that $\pm 1=c e^{\prime}-e c^{\prime}$, so $\operatorname{gcd}\left(c, c^{\prime}\right)=1$. Note that we cannot have $c=c^{\prime}=1$ as otherwise some nontrivial surgery on a hyperbolic knot in $S^{3}$ would yield $S^{3}$, contrary to [19]. Thus $c \neq c^{\prime}$, so the proposition holds when $Z(K)$ acts freely on $S^{3}$.

Suppose next that $Z(K)$ does not act freely on $S^{3}$. By Proposition 5.7, $K$ is a $(p, q ; a)$-unwrapped Berge-Gabai knot and $K^{\prime}$ is a $\left(p^{\prime}, q^{\prime} ; a\right)$-unwrapped BergeGabai knot where $p$ and $p^{\prime}$ are coprime by Lemma 5.6 and $a>1$. Since $|Z(K)|=a p$ and $\left|Z\left(K^{\prime}\right)\right|=a p^{\prime}$, it follows that $|Z(K)| \neq\left|Z\left(K^{\prime}\right)\right|$ unless $p=p^{\prime}=1$. Assume $p=p^{\prime}=1$. There is a Heegaard splitting $|\mathcal{L}(1, q ; a)|=V_{1} \cup V_{2}$ where the singular set of $\mathcal{L}(1, q ; a)$ is the core $C_{2}$ of $V_{2}$ and a hyperbolic Berge-Gabai knot $\bar{K} \subset V_{1} \subset \mathcal{L}(1, q ; a)$ such that $K$ is the inverse image of $\bar{K}$ in $S^{3}$. Since $C_{2}$ is unknotted in $|\mathcal{L}(1, q ; a)| \cong S^{3}$, Corollary 3.5 of [14] implies that its image is knotted in $|\mathcal{L}(1, q ; a)| \cong S^{3}$. But this contradicts the fact that the image of $C_{2}$ in $\left|\mathcal{L}\left(1, q^{\prime} ; a\right)\right|$ is the core of a Heegaard solid torus. Hence we cannot have $p=p^{\prime}=1$. This completes the proof that $Z(K)$ and $Z\left(K^{\prime}\right)$ have distinct orders and therefore that $K$ and $K^{\prime}$ have distinct volumes.

Since mutant hyperbolic knots have the same volume, $K$ and $K^{\prime}$ cannot be mutant. Similarly hyperbolic knots which are mirror images of each other have the same volume so as $K^{\prime} \neq K, K^{\prime}$ cannot be the mirror image of $K$.

\section{Fibred knots}

In this section we prove that any hyperbolic knot without hidden symmetries and with $|\mathcal{C C}(K)| \geq 2$ is fibred (Theorem 1.7(1)).

We divide the proof of Theorem 1.7(1) into two cases according to whether $K$ is periodic or not.

\section{1 $K$ is periodic}

Here we prove a fibring theorem for 1-bridge braid exteriors and apply it to deduce the periodic case of Theorem 1.7(1).

Theorem 6.1 Let $K$ be a 1-bridge braid on $n$ strands in a solid torus $V$. For any essential simple closed curve $C$ on $\partial V$ whose algebraic winding number in $V$ is coprime to $n$ there is a locally trivial fibring of the exterior of $K$ in $V$ by surfaces whose intersection with $\partial V$ has $n$ components, each a curve parallel to $C$. 


\section{Corollary 6.2 An unwrapped Berge-Gabai knot is a fibred knot.}

Proof of Corollary 6.2 Let $K$ be an unwrapped Berge-Gabai knot in $S^{3}$. Then $K$ is the inverse image in $S^{3}$ of a Berge-Gabai knot $\bar{K} \subset \mathcal{L}(p, q ; a)$ of winding number $n$, say, under the universal cover $S^{3} \rightarrow \mathcal{L}(p, q ; a)$. Thus there is a genus one Heegaard splitting $V_{1} \cup V_{2}$ of $|\mathcal{L}(p, q ; a)|$ such that $\bar{K}$ is a Berge-Gabai knot of winding number $n$ in $V_{1}$ and $\Sigma(\mathcal{L}(p, q ; a))$ is a closed submanifold of the core $C_{2}$ of $V_{2}$. As $|\mathcal{L}(p, q ; a)|=L(p, q)$, the algebraic intersection number of a meridian curve of $V_{1}$ with one of $V_{2}$ is $\pm p$. By definition, $\operatorname{gcd}(p, n)=1$, so Theorem 6.1 implies that there is a locally trivial fibring of the exterior of $\bar{K}$ by surfaces which intersect $\partial V_{1}$ in curves parallel to the meridian of $V_{2}$. Therefore we can extend the fibration over the exterior of $K$ in $L(p, q)=|\mathcal{L}(p, q ; a)|$ in such a way that it everywhere transverse to $\Sigma(\mathcal{L}(p, q ; a))$. Hence the fibration lifts to a fibring of the exterior of $K$.

Proof of Theorem 6.1 Let $K$ be the closed 1-bridge braid contained in the interior of a solid torus $V$ determined by the three parameters:

- $n$, the braid index of $K$.

- $b$, the bridge index of $K$.

- $t$, the twisting number of $K$.

See Gabai [14] for an explanation of these parameters and Figure 1 for an example. (Our conventions differ from those of [14] by mirroring and changing orientation. This modification is convenient for presenting the knot's fundamental group.)

For $a \in \mathbb{Z}$, let $\bar{a}$ denote the $(\bmod n)$ equivalence class of $a$. Number the braid's strands successively $\overline{0}$ to $\overline{n-1}$ and let $\sigma_{i}$ denote the $i$-th elementary braid in which the $i$-th strand passes over the $(i+1)-$ st. The braid associated to $K$ has the following form: $\beta(K)=\sigma_{b-1} \cdots \sigma_{0} \delta^{t}$ where $\delta=\sigma_{n-2} \cdots \sigma_{0}$ is the positive $2 \pi / n$ twist. Denote by $\pi$ the permutation of $\mathbb{Z} / n$ determined by $\beta(K)$. It has the following simple form:

$$
\pi(\bar{a})= \begin{cases}\overline{a+t+1} & \text { if } 0 \leq a<b, \\ \bar{t} & \text { if } a=b, \\ \overline{a+t} & \text { if } b<a<n,\end{cases}
$$

for some $a \in \bar{a}$. As $K$ is a knot, $\pi$ is an $n$-cycle.

Let $T_{1}=\partial V$ and $T_{2}=\partial N(K)$ the boundary of a closed tubular neighborhood of $K$ in $\operatorname{int}(V)$. There is a meridian class $\mu_{1} \in H_{1}\left(T_{1}\right)$ well-defined up to \pm 1 and represented 


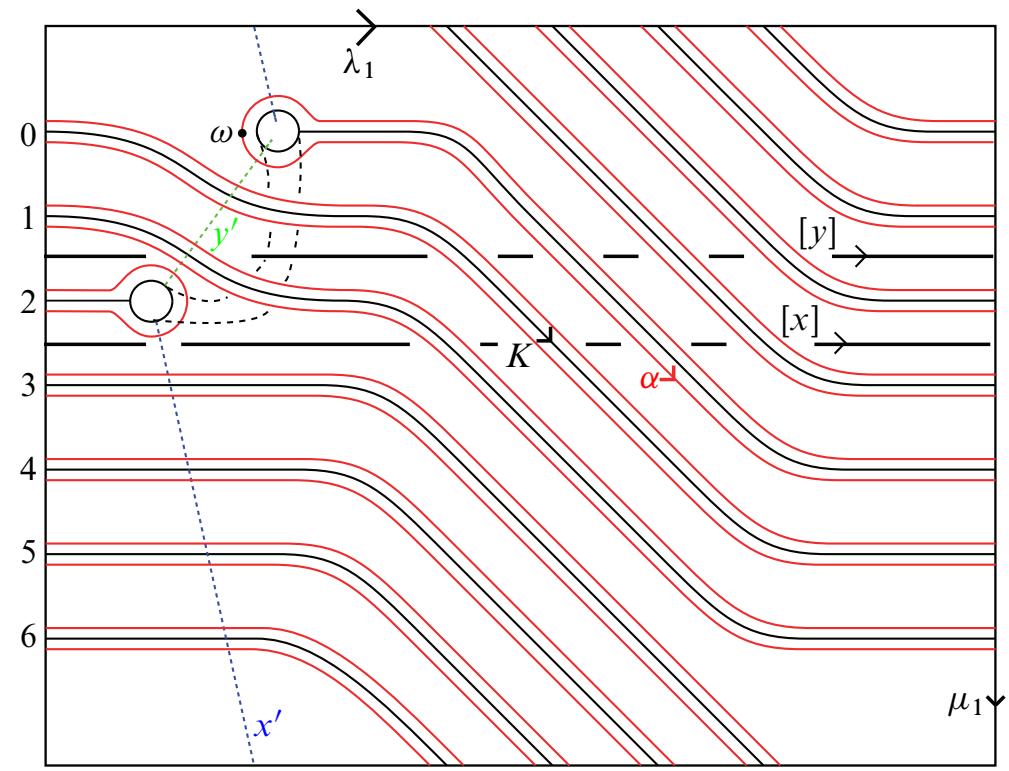

Figure 1. The Fintushel-Stern knot $(n=7, b=2, t=4)$. The curve $x^{\prime}$ is obtained from the arc labeled $x^{\prime}$ by closing it in the boundary of the tunnel with an arc parallel to the bridge and $y^{\prime}$ is obtained similarly by closing the arc $y^{\prime}$ in the boundary of the tunnel. Here $R$ is the word $y x y x x y x x y^{-1} x^{-1} y^{-1} x^{-1} x^{-1} y^{-1} x^{-1} x^{-1}$.

by the boundary of a meridian disk of $V_{1}$. Let $\lambda_{1} \in H_{1}\left(T_{1}\right)$ be any class which forms a basis of $H_{1}\left(T_{1}\right)$ with $\mu_{1}$. Then $\lambda_{1}$ generates $H_{1}(V)$.

Let $M$ denote the exterior of $K$ in $V$ and fix an essential simple closed curve $C$ on $\partial V$. We are clearly done if $C$ is a meridian curve of $V$, so assume that this is not the case. Then we can orient $C$ and find coprime integers $p \geq 1, q$ so that

$$
[C]=q \mu_{1}+p \lambda_{1} \in H_{1}\left(T_{1}\right) \text {. }
$$

Note that $p$ is the algebraic winding number of $C$ in $V$. Assuming that $\operatorname{gcd}(p, n)=1$ we must show that there is a locally trivial fibring of $M$ by surfaces which intersect $\partial V$ in curves parallel to $C$. The tools we use to prove this are Brown's theorem [8] and Stallings' fibration criterion [44]. See also Ozsváth and Szabó [36] where a similar argument is invoked; our proof is only slightly more involved. Brown's theorem gives necessary and sufficient conditions under which a homomorphism from a two-generator one-relator group to $\mathbb{Z}$ has finitely generated kernel and Stallings' theorem produces a fibration of a 3-manifold given such a homomorphism of its fundamental group. More precisely: 
Theorem 6.3 [8, Theorem 4.3 and Proposition 3.1] Let $G=\langle x, y: R\rangle$ be a twogenerator one-relator group with $R=R_{1} R_{2} \cdots R_{m}, R_{i} \in\left\{x, x^{-1}, y, y^{-1}\right\}$, a cyclically reduced and nontrivial relator. Let $S_{1}, \ldots, S_{m}$ be the proper initial segments of the relator $R$, ie $S_{i}=R_{1} \cdots R_{i-1}$. Finally let $\varphi: G \rightarrow \mathbb{R}$ be a nonzero homomorphism. If $\varphi(x) \neq 0$ and $\varphi(y) \neq 0$, then $\operatorname{ker}(\varphi)$ is finitely generated if and only if the sequence $\left\{\varphi\left(S_{i}\right)\right\}_{i=1}^{m}$ assumes its maximum and minimum values exactly once.

It is easy to see that the exterior $M$ of $K$ is homeomorphic to a genus 2 handlebody with a 2-handle attached to it. Start with a solid torus $U^{\prime} \subset \operatorname{int}(V)$ obtained by removing a small open collar of $T_{1}$ in $V$. Denote $\partial U^{\prime}$ by $T_{3}$. As $K$ is 1 -bridge, it can be isotoped into $U^{\prime}$ so that the bridge is a properly embedded arc and its complement, $\gamma$ say, is contained in $T_{3}$. Fix a disk neighborhood $D \subset T_{3}$ of $\gamma$ and let $\alpha=\partial D$. Let $U$ be the exterior of the bridge in $U^{\prime}$, a genus two handlebody. We can assume that $T_{3} \backslash \partial U \subset \operatorname{int}(D)$ and therefore $\alpha \subset \partial U$. By construction, $\alpha$ bounds a 2-disk properly embedded in $\overline{V \backslash U}$ (ie a copy of $D$ isotoped rel $\partial D$ into $\overline{V \backslash U}$ ). It is easy to see that $M$ is a regular neighborhood of the union of $U$ and this disk.

The fundamental group of $U$ is free on two generators $x, y$ represented by two curves in $T_{3}$ representing $\lambda_{1}$. (See Figure 1.) There are a pair of dual curves $x^{\prime}, y^{\prime} \subset \partial U$ to these generators. This means that

- $\quad x^{\prime}$ and $y^{\prime}$ bound disks in $U$.

- $x$ intersects $x^{\prime}$ transversely in one point and is disjoint from $y^{\prime}$.

- $y$ intersects $y^{\prime}$ transversely in one point and is disjoint from $x^{\prime}$.

See Figure 1. The word $R \in \pi_{1}(U)$ in $x, y$ represented by the curve $\alpha$ can be read off in the usual way: each signed intersection of $\alpha$ with $x^{\prime}$, resp. $y^{\prime}$, contributes $x^{ \pm 1}$, resp. $y^{ \pm 1}$, while traveling around $\alpha$.

We introduce the auxiliary function $f: \mathbb{Z} / n \backslash\{\bar{b}\} \rightarrow\{x, y\}$ given by

$$
f(\bar{a})= \begin{cases}y & \text { if } 0 \leq a<b, \\ x & \text { if } b<a<n,\end{cases}
$$

for some $a \in \bar{a}$. Let $w_{j}=f\left(\pi^{j}(\bar{b})\right)$ and consider the word $w=w_{1} w_{2} \cdots w_{n-1}$. Then $R=y w x y^{-1} w^{-1} x^{-1}$. To see this, start with $y$ from the base point $\omega$ (cf Figure 1); then follow the knot until the $b$ strand, which contributes $w$; then turn at the lower foot of the handle, which contributes $x y^{-1}$; then walk along the knot in the opposite direction until the strand $b$ is reached, which contributes $w^{-1}$; then close by passing $x^{\prime}$, which contributes to the final $x^{-1}$. Notice that $R$ is cyclically reduced. It follows that

$$
\pi_{1}(M)=\left\langle x, y: y w x y^{-1} w^{-1} x^{-1}\right\rangle .
$$


Let $\mu_{2} \in H_{1}\left(T_{2}\right)$ be a meridional class of $K$. The reader will verify that we can choose the longitudinal class $\lambda_{1}$ for $V$, a longitudinal class $\lambda_{2} \in H_{1}\left(T_{2}\right)$ for $K$, and possibly replace $\mu_{1}$ by $-\mu_{1}$ so that in $H_{1}(M)$,

- $n \lambda_{1}=\lambda_{2}$.

- $\mu_{1}=n \mu_{2}$.

- $\left[y x^{-1}\right]=\mu_{2}$ (ie $\left[y x^{-1}\right]$ is represented by a meridian of $K$ at the bridge).

- $\lambda_{1}+t \mu_{2}=[x]$ (ie $\lambda_{1}$ and $[x]$ cobound an annulus in $V$ which $K$ punctures $t$ times).

Consider the homomorphism $\pi_{1}(U) \rightarrow \mathbb{Z}$ which sends $x$ to $p t-n q \neq 0$ and $y$ to $p t-n q+p \neq 0$. Since the exponent sum of both $x$ and $y$ in $R$ is zero, it induces a homomorphism $\varphi: \pi_{1}(M) \rightarrow \mathbb{Z}$. Since $\operatorname{gcd}(p, n q)=1, \varphi$ is surjective. From the above, it can then be verified that $\varphi\left(\lambda_{1}\right)=-n q$ and $\varphi\left(\mu_{1}\right)=n p$. Hence $\varphi\left(\mu_{1}^{q} \lambda_{1}^{p}\right)=0$.

Lemma 6.4 Let $S_{1}, S_{2}, \ldots, S_{2 n+2}$ be the proper initial segments of the relator $R=y w x y^{-1} w^{-1} x^{-1}=R_{1} R_{2} \cdots R_{2 n+2}$ where $R_{i} \in\left\{x, x^{-1}, y, y^{-1}\right\}$. Then the sequence $\left\{\varphi\left(S_{i}\right)\right\}_{i=1}^{2 n+2}$ achieves its maximum and minimum values exactly once.

Proof By construction, $\varphi(x) \neq 0, \varphi(y) \neq 0$, and $\varphi(y)>\varphi(x)$. The conclusion of the lemma is easily seen to hold when $\varphi(x)$ and $\varphi(y)$ have the same sign, so assume that $\varphi(x)<0<\varphi(y)$.

Set $S=\max \left\{\varphi\left(S_{i}\right): 1 \leq i \leq 2 n+2\right\}$ and $s=\min \left\{\varphi\left(S_{i}\right): 1 \leq i \leq 2 n+2\right\}$.

Since $\varphi(x)<0<\varphi(y)$ we have

$$
\left\{\begin{array}{l}
s \leq \varphi\left(S_{n+2}\right)<\varphi\left(S_{n+1}\right)<\varphi\left(S_{n}\right) \leq S, \\
s \leq \varphi\left(S_{n+i}\right)=\varphi\left(S_{n-i+2}\right)+\varphi(x)-\varphi(y)<\varphi\left(S_{n-i+2}\right) \leq S \text { for } 3 \leq i \leq n+1, \\
s \leq \varphi\left(S_{2 n+1}\right)=\varphi\left(S_{2 n+2}\right)+\varphi(x)<\varphi\left(S_{2 n+2}\right)=0<\varphi(y)=\varphi\left(S_{1}\right) \leq S .
\end{array}\right.
$$

Thus the maxima of $\left\{\varphi\left(S_{i}\right)\right\}_{i=1}^{2 n+2}$ can only occur in the sequence $\varphi\left(S_{1}\right), \varphi\left(S_{2}\right), \ldots$, $\varphi\left(S_{n}\right)$ and the minima in $\varphi\left(S_{n+2}\right), \varphi\left(S_{n+3}\right), \ldots, \varphi\left(S_{2 n+1}\right)$.

We look at the maxima of $\left\{\varphi\left(S_{i}\right)\right\}_{i=1}^{2 n+2}$ first. Suppose that $1 \leq l<r \leq n$. We claim that $\varphi\left(R_{l+1}\right)+\cdots+\varphi\left(R_{r}\right) \not \equiv 0(\bmod n)$. If so, $\varphi\left(S_{l}\right) \neq \varphi\left(S_{r}\right)$ and therefore $S$ occurs precisely once amongst the values $\left\{\varphi\left(S_{i}\right)\right\}_{i=1}^{n}$.

Let $\bar{\varphi}$ be the reduction of $\varphi$ modulo $n$. Since $\operatorname{gcd}(p, n)=1$, we can define

$$
\widehat{\varphi}=\bar{p}^{1} \bar{\varphi}: \pi_{1}(M) \rightarrow \mathbb{Z} / n .
$$


Then $\hat{\varphi}(x)=\bar{t}$ and $\hat{\varphi}(y)=\overline{t+1}$ and therefore

$$
\hat{\varphi}(f(\bar{a}))=\pi(\bar{a})-\bar{a}
$$

for all $a \in \mathbb{Z} / n \backslash\{\bar{b}\}$. Hence $\hat{\varphi}\left(R_{l+1}\right)+\cdots+\hat{\varphi}\left(R_{r}\right)=\hat{\varphi}\left(w_{l}\right)+\cdots+\hat{\varphi}\left(w_{r-1}\right)=$ $\hat{\varphi}\left(f\left(\pi^{l}(\bar{b})\right)\right)+\cdots+\hat{\varphi}\left(f\left(\pi^{r-1}(\bar{b})\right)\right)=\left(\pi^{l+1}(\bar{b})-\pi^{l}(\bar{b})\right)+\cdots+\left(\pi^{r}(\bar{b})-\pi^{r-1}(\bar{b})\right)=$ $\pi^{r}(\bar{b})-\pi^{l}(\bar{b})$. Since $\pi$ is an $n$-cycle and $1 \leq l<r \leq n$ we see that $\pi^{r}(\bar{b}) \neq \pi^{l}(\bar{b})$. It follows that $\varphi\left(R_{l+1}\right)+\cdots+\varphi\left(R_{r}\right) \not \equiv 0(\bmod n)$.

The uniqueness of the minimum follows along the same lines. We saw above that the minima of $\left\{\varphi\left(S_{i}\right)\right\}_{i=1}^{2 n+2}$ only occur in $\varphi\left(S_{n+2}\right), \varphi\left(S_{n+3}\right), \ldots, \varphi\left(S_{2 n+1}\right)$. As before, $\varphi\left(R_{l+1}\right)+\cdots+\varphi\left(R_{r}\right) \not \equiv 0(\bmod n)$ for all $n+2 \leq l<r \leq 2 n+1$ and therefore $\varphi\left(S_{n+2}\right), \varphi\left(S_{n+3}\right), \ldots, \varphi\left(S_{2 n+1}\right)$ are pairwise distinct. This implies the desired conclusion.

We can now complete the proof of Theorem 6.1. The previous lemma couples with Theorem 6.3 to show that the kernel of $\varphi$ is finitely generated. Stallings' fibration criterion [44] implies that $M$ admits a locally trivial surface fibration with fibre $F$ such that $\pi_{1}(F)=\operatorname{ker}(\varphi)$. Since $\varphi\left(\mu_{1}\right)=n p \neq 0$ while $\varphi\left(\mu_{1}^{q} \lambda_{1}^{p}\right)=0, \operatorname{ker}\left(\left.\varphi\right|_{\pi_{1}\left(T_{1}\right)}\right)$ is the infinite cyclic subgroup of $\pi_{1}\left(T_{1}\right)$ generated by $[C]$. Hence the fibration meets $T_{1}$ in curves parallel to $C$. To complete the proof, we must show that the intersection of a fibre $F$ with $T_{1}$ has $n$ components.

To that end, note that as $\varphi$ is surjective we can orient $F$ so that for each $\zeta \in H_{1}(M)$ we have $\varphi(\zeta)=\zeta \cdot[F]$. Let $\phi_{1} \in H_{1}(M)$ be the class represented by the cycle $F \cap T_{1}$ with the induced orientation. Clearly, $\phi_{1}= \pm\left|F \cap T_{1}\right|[C]$. Since $\varphi\left(\lambda_{1}\right)=-n q$ and $\varphi\left(\mu_{1}\right)=n p, \varphi\left(\pi_{1}\left(T_{1}\right)\right)=n \mathbb{Z}$. Thus if $\zeta \in H_{1}(M)$ is represented by a dual cycle to $[C]$ on $T_{1}$, then

$$
n=\varphi(\zeta)=\zeta \cdot[F]=\left|\zeta \cdot \phi_{1}\right|=|| F \cap T_{1}|\zeta \cdot[C]|=\left|F \cap T_{1}\right| .
$$

This completes the proof.

Proof of Proposition 1.6 Let $K$ be a hyperbolic 1-bridge braid on $n$ strands in a solid torus $V$. We use the notation developed in the proof of Theorem 6.1. In particular, $M$ is the exterior of $K$ in $V$ and $H_{1}(M) \cong \mathbb{Z} \oplus \mathbb{Z}$ with basis $\lambda_{1}, \mu_{2}$. By construction there are classes $\xi_{1}, \xi_{2} \in H_{2}(M, \partial M)$ such that if $\partial: H_{2}(M, \partial M) \rightarrow H_{1}(\partial M)$ is the connecting homomorphism, then $\partial \xi_{1}=\mu_{1}-n \mu_{2}$ and $\partial \xi_{2}=n \lambda_{1}-\lambda_{2}$. Since $\left|\lambda_{1} \cdot \xi_{j}\right|=\delta_{1 j}$ and $\left|\mu_{2} \cdot \xi_{j}\right|=\delta_{2 j},\left\{\xi_{1}, \xi_{2}\right\}$ is a basis for $H_{2}(M, \partial M) \cong H^{1}(M) \cong \mathbb{Z} \oplus \mathbb{Z}$.

Consider the homomorphism $\psi$ given by the composition $H_{2}(M, \partial M) \stackrel{\partial}{\longrightarrow} H_{1}(\partial M)=$ $H_{1}\left(T_{1}\right) \oplus H_{1}\left(T_{2}\right) \rightarrow H_{1}\left(T_{1}\right)$. Then $\psi\left(a \xi_{1}+b \xi_{2}\right)=a \mu_{1}+n b \lambda_{1}$, and therefore $\psi$ is injective. 
Let $p, q$ be coprime integers such that $\operatorname{gcd}(n, p)=1$. According to Theorem 6.1, there is a fibre $F$ in $M$ which can be oriented so that $\psi([F])=\left[F \cap T_{1}\right]=n q \mu_{1}+n p \lambda_{1}=$ $\psi\left(n q \xi_{1}+p \xi_{2}\right)$. Hence $[F]=n q \xi_{1}+p \xi_{2}$ so that $n q \xi_{1}+p \xi_{2}$ is a fibre class in $\mathrm{H}_{2}(M, \partial M)$.

Fix coprime integers $a, b$ and consider the class $\xi=a \xi_{1}+b \xi_{2}$. The proposition will follow if we can show that the projective class of $\xi$ can be arbitrarily closely approximated by fibre classes [45, Theorem 2]. By the previous paragraph $\xi$ is a fibre class when $a=0$, so suppose this is not the case. It suffices to show that $b / a=\lim _{m}\left(b_{m} / a_{m}\right)$ where $a_{m} \xi_{1}+b_{m} \xi_{2}$ are fibre classes. This is easy to verify: for each integer $m>0$ set $p_{m}=n m b a+1$ and $q_{m}=m b^{2}$. Then $\operatorname{gcd}\left(p_{m}, n q_{m}\right)=1$ and from the previous paragraph we see that $n q_{m} \xi_{1}+p_{m} \xi_{2}$ is a fibre class. Finally, $\lim _{m}\left(n q_{m} / p_{m}\right)=b / a$, which completes the proof.

\section{2 $K$ is not periodic}

In this case, $Z(K)$ is generated by a free symmetry of the pair $\left(S^{3}, K\right)$. Then $\mathcal{Z}_{K}$ is a complete hyperbolic 3-manifold with a torus cusp and $\mathcal{Z}_{K}(r(K))=\mathcal{L}_{K}$ is a lens space $L(p, q)$. The image $\bar{K}$ of $K$ in $\mathcal{Z}_{K}(r(K))$ is primitive, since its preimage in the universal cover $S^{3}$ has one component. Since $|\mathcal{C C}(K)|>1$, Proposition 4.13 shows that there is another slope $r^{\prime}$ in the torus cusp of $\mathcal{Z}_{K}$ such that $\mathcal{Z}_{K}\left(r^{\prime}\right)$ is a lens space $L\left(p^{\prime}, q^{\prime}\right)$. The following key result has been explained to us by Jake Rasmussen.

Theorem 6.5 Let $K$ be a primitive knot in $Y=L(p, q)$ which admits a nontrivial lens space surgery. Then $K$ is fibred.

This theorem shows that $\mathcal{Z}_{K}$ is a surface bundle over the circle, and since there is an unbranched cover $S^{3} \backslash K \rightarrow \mathcal{Z}_{K}, K$ is a fibred knot. Many, though not all, of the elements of the proof of Theorem 6.5 are contained in Rasmussen [38]. Owing to its importance to this paper, we include a proof here.

Proof of Theorem 6.5 The analogous result is known to be true for knots in the 3-sphere [30]: If a knot $K \subset S^{3}$ has a lens space surgery, then $K$ is fibred. The proof of Ni's result uses the Heegaard Floer homology package developed by Ozsváth and Szabó in $[35 ; 33]$ and extended to sutured manifolds by Ni and Juhasz [30; 22]. The essential property of lens spaces which is invoked is that they are L-spaces, which are rational homology spheres with Heegaard Floer homology as simple as possible (cf [36]). Our situation is similar in that both the initial and the surgered manifold are L-spaces, and our proof follows that of the $S^{3}$ case. 
We first note that if $L(p, q) \backslash K$ is Seifert-fibred and $K$ is primitive, then $N \cong$ $L(p, q) \backslash N(K)$ is cyclically covered by a knot complement $M$ in $S^{3}$ which must also be Seifert-fibred. Then $M$ must be a torus knot complement, which fibres over $S^{1}$. Since $H_{2}(M, \partial M) \cong H_{2}(N, \partial N) \cong \mathbb{Z}$, the preimage of a minimal genus representative of the generator of $\mathrm{H}_{2}(N, \partial N)$ is a union of surface fibres of $M$, and the covering action permutes these fibres. The region between a fibre and its image under a generator for the covering group is a fundamental domain of this action, so $N$ is fibred.

For the remainder of the proof we assume that $L(p, q) \backslash K$ is not Seifert-fibred.

Let $\mu_{K}$ denote the meridional slope of $K$ and $\lambda \neq \mu_{K}$ a slope whose associated surgery yields a lens space. By the cyclic surgery theorem $[10], \Delta\left(\lambda, \mu_{K}\right)=1$, so any representative curve for $\lambda$ runs parallel to $K$.

We find it convenient to use the notation from [32] even though it is somewhat different from that used elsewhere in the paper. We review this notation here.

We use $\bar{K}$ to denote the knot $K$ with a choice of orientation. Dehn surgery on $K$ with slope $\lambda$ will be written $Y_{\lambda}(K)$. In [32], Ozsváth and Szabó compute the Heegaard Floer homology of manifolds obtained by surgery on knots in rational homology spheres in terms of the knot filtration on the chain complex whose homology is the Heegaard Floer homology of the ambient manifold. Based on this, Rasmussen computes the knot Floer homology of knots in lens spaces which admit longitudinal surgeries that produce integral L-spaces [38, Lemma 4.7]. The strategy here is to do the same calculation for knots admitting longitudinal surgeries which produce rational L-spaces and then to pass to the Floer homology of a certain sutured manifold.

There is a doubly pointed Heegaard diagram $(\Sigma, \boldsymbol{\alpha}, \boldsymbol{\beta}, w, z)$ which determines $(Y, \bar{K})$, from which Ozsváth and Szabó construct a chain complex $C F K^{\infty}(\Sigma, \boldsymbol{\alpha}, \boldsymbol{\beta}, w, z)$ as follows. The generating set is $\left\{[\mathbf{x}, i, j]: \mathbf{x} \in \mathbb{T}_{\alpha} \cap \mathbb{T}_{\beta}, i, j \in \mathbb{Z}\right\}$ where $\mathbb{T}_{\alpha}$ and $\mathbb{T}_{\beta}$ are two totally real tori in the symmetric product $\operatorname{Sym}^{g}(\Sigma)$ which is endowed with an almost complex structure. The differential counts certain pseudoholomorphic disks connecting the generators with the boundary mapping to $\mathbb{T}_{\alpha} \cup \mathbb{T}_{\beta}$. The two basepoints $w$ and $z$ give rise to codimension 2 submanifolds $\{w\} \times \operatorname{Sym}^{g-1}(\Sigma)$, resp. $\{z\} \times \operatorname{Sym}^{g-1}(\Sigma)$. The indices $i, j$ are employed in order to keep track of the intersection of the holomorphic disks with the two submanifolds above. More precisely,

$$
\partial^{\infty}[\mathbf{x}, i, j]=\sum_{\mathbf{y} \in \mathbb{T}_{\alpha} \cap \mathbb{T}_{\beta}} \sum_{\phi \in \pi_{2}(\mathbf{x}, \mathbf{y}), \mu(\phi)=1} \#\left(\frac{M(\phi)}{\mathbb{R}}\right) \cdot\left[\mathbf{y}, i-n_{w}(\phi), j-n_{z}(\phi)\right],
$$

where $\pi_{2}(\mathbf{x}, \mathbf{y})$ denotes the homotopy class of disks connecting $\mathbf{x}$ and $\mathbf{y}, \mu(\phi)$ is the Maslov index of $\phi, \#(M(\phi) / \mathbb{R})$ is the count of holomorphic representatives of $\phi$, 
$n_{w}(\phi)=\# \phi \cap\left(\{w\} \times \operatorname{Sym}^{g-1}(\Sigma)\right)$, and similarly for $n_{z}(\phi)$. Note that $n_{w}(\phi) \geq 0$, $n_{z}(\phi) \geq 0$ since the submanifolds involved are almost complex. Therefore the $i, j$ indices define a $\mathbb{Z} \oplus \mathbb{Z}$ filtration on $C F K^{\infty}(Y, \bar{K})$.

The nontriviality of $\phi \in \pi_{2}(\mathbf{x}, \mathbf{y})$ is homologically obstructed and as consequence the complex $C F K^{\infty}(Y, \bar{K})$ splits into summands which are in bijection with $\operatorname{Spin}^{c}$ structures on $Y$. Following Turaev [46] $\operatorname{Spin}^{c}$ structures can be seen as homology classes of nonvanishing vector fields and they form an affine space over $H^{2}(Y)$. From the combinatorics of the Heegaard diagram one can construct a function $\mathfrak{s}_{w}: \mathbb{T}_{\alpha} \cap \mathbb{T}_{\beta} \rightarrow$ $\operatorname{Spin}^{c}(Y)$ which sends an intersection point $\mathbf{x}$ to the homology class of a vector field. There is also a relative version, $\operatorname{Spin}^{c}(Y, K)$, which consists of homology classes of vector fields on $Y \backslash N(K)$ which point outwards at the boundary; one has an analogous map $\mathfrak{s}_{w, z}: \mathbb{T}_{\alpha} \cap \mathbb{T}_{\beta} \rightarrow \underline{\operatorname{Spin}^{c}}(Y, K)$ [32, Section 2.4]. $\underline{\operatorname{Spin}^{c}}(Y, K)$ is an affine space over $H^{2}(Y, K)$.

One can extend a vector field on $Y \backslash N(K)$ to a vector field on $Y$ such that the (oriented) knot is a closed trajectory. This gives rise to a map $G_{Y, \bar{K}}: \operatorname{Spin}^{c}(Y, K) \rightarrow$ $\operatorname{Spin}^{c}(Y)$ which is equivariant with respect to the action of $H^{2}(Y, K): \overline{G_{Y, \bar{K}}}(\xi+k)=$ $G_{Y, \bar{K}}(\xi)+\iota^{*}(k)$. where $\iota^{*}: H^{2}(Y, K) \rightarrow H^{2}(Y)$ is the natural map induced by inclusion. Moreover, given $\xi, \eta \in \operatorname{Spin}^{c}(Y, K), G_{Y, \bar{K}}(\xi)=G_{Y, \bar{K}}(\eta)$ if and only if $\xi=\eta+n \cdot \operatorname{PD}[\mu]$ for some $n \in \mathbb{Z}$ where $\mu$ is a meridian of $K$. There is an analogous map $G_{Y_{\lambda}(K), \bar{K}}: \underline{\operatorname{Spin}^{c}}(Y, K) \rightarrow \operatorname{Spin}^{c}\left(Y_{\lambda}(K)\right)$ which extends the vector fields on $Y \backslash N(K)$ to $Y_{\lambda} \overline{(K)}$ such that the induced knot $K^{\prime}$ in the surgered manifold becomes a trajectory with the orientation inherited from $K$. See [32, Section 6].

For $\mathbf{x}, \mathbf{y} \in \mathbb{T}_{\alpha} \cap \mathbb{T}_{\beta}$ such that there exists $\phi \in \pi_{2}(\mathbf{x}, \mathbf{y})$, we have: $\mathfrak{s}_{w, z}(\mathbf{x})-\mathfrak{s}_{w, z}(\mathbf{y})=$ $\left(n_{z}(\phi)-n_{w}(\phi)\right) \cdot \operatorname{PD}[\mu]\left[32\right.$, Lemma 2.1]. This splits $C F K^{\infty}(Y, \bar{K})$ into various summands: Fix $\xi \in \operatorname{Spin}^{c}(Y, K)$. The subgroup

$$
C_{\xi}:=\left\{[\mathbf{x}, i, j] \in C F K^{\infty}(Y, \bar{K}): \mathfrak{s}_{w, z}(\mathbf{x})+(i-j) \cdot \operatorname{PD}[\mu]=\xi\right\}
$$

becomes a subcomplex of $C F K^{\infty}(Y, \bar{K})$.

Ozsváth and Szabó consider the induced complexes $A_{\xi}(Y, \bar{K})$ and $B_{\xi}(Y, \bar{K})$ as ingredients in the Morse surgery formula: The complex $A_{\xi}(Y, \bar{K}):=C_{\xi}\{\max (i, j)=0\}$ with the induced differential from the complex $C F K^{\infty}(Y, \bar{K})$ computes the Heegaard Floer homology of large enough integral surgeries on $K$ in a particular $\operatorname{Spin}^{c}$ structure [32, Theorem 4.1]. Since $\chi(\widehat{H F}(M, \mathfrak{s}))=1$ for all $\mathfrak{s} \in \operatorname{Spin}^{c}(M)$ where $M$ is any rational homology sphere [34, Theorem 5.1] and framed surgeries on $K$ are rational homology spheres, we have that $\operatorname{rank}\left(H_{*}\left(A_{\xi}(Y, \bar{K})\right)\right) \geq 1$.

By definition, $B_{\xi}(Y, \bar{K}):=C_{\xi}\{i=0\}$ computes $\widehat{H F}\left(Y, G_{Y, \bar{K}}(\xi)\right)$. In addition, $C_{\xi}\{j=0\}$ is identified with $B_{\xi+\mathrm{PD}\left[K_{\lambda}\right]}$, where $K_{\lambda}$ is the knot $K$ pushed off inside 
$Y \backslash N(K)$ along the framing $\lambda$. See [32, Proposition 3.2] for an explanation of the grading shift.

Consider also the maps $v_{\xi}$, resp. $h_{\xi}$, the natural projections $C_{\xi}\{\max (i, j)=0\} \rightarrow$ $C_{\xi}\{i=0\}$, resp. $C_{\xi}\{\max (i, j)=0\} \rightarrow C_{\xi}\{j=0\}$.

By [32, Theorem 4.1] they are identified with the induced maps in $\widehat{H F}$ by the natural cobordism $W_{m}^{\prime}(K)$ between $Y_{\lambda}(K)$ and $Y$ equipped with corresponding $\operatorname{Spin}^{c}$ structures.

We can now state the surgery formula for the "hat" version of Heegaard Floer homology. This corresponds to taking $\delta=0$ in [32, Theorem 6.4]. See [32, Section 2.8] for an explanation. We therefore drop the $\delta$ index in what follows:

Theorem 6.6 [32, Theorem 6.4] Fix a $\operatorname{Spin}^{c}$ structure $\mathfrak{s} \in \operatorname{Spin}^{c}\left(Y_{\lambda}(K)\right)$. Then

$$
\widehat{H F}\left(Y_{\lambda}(K), \mathfrak{s}\right) \cong H_{*}\left(\operatorname{Cone}\left(D_{\mathfrak{s}}: \mathbb{A}_{\mathfrak{s}}(Y, K) \rightarrow \mathbb{B}_{\mathfrak{s}}(Y, K)\right)\right)
$$

where

$$
\begin{aligned}
& \mathbb{A}_{\mathfrak{s}}(Y, K)=\bigoplus_{\left\{\xi \in \underline{\operatorname{Spin}^{c}}\left(Y_{\lambda}(K), K\right) \mid G_{Y_{\lambda}(K), \bar{K}}(\xi)=\mathfrak{s}\right\}} A_{\xi}(Y, \bar{K}), \\
& \mathbb{B}_{\mathfrak{s}}(Y, K)=\bigoplus_{\left\{\xi \in \underline{\operatorname{Spin}^{c}}\left(Y_{\lambda}(K), K\right) \mid G_{Y_{\lambda}(K), \bar{K}}(\xi)=\mathfrak{s}\right\}} B_{\xi}(Y, \bar{K}) .
\end{aligned}
$$

The map $D_{\mathfrak{s}}$ is defined as

$$
D_{\mathfrak{s}}\left(\left\{a_{\xi}\right\}_{\xi \in G_{Y_{\lambda}(K), \bar{K}}^{-1}(\mathfrak{s})}\right)=\left\{b_{\xi}\right\}_{\xi \in G_{Y_{\lambda}(K), \bar{K}}^{-1}(\mathfrak{s})}
$$

with $b_{\xi}=h_{\xi-\mathrm{PD}\left[K_{\lambda}\right]}\left(a_{\xi-\mathrm{PD}\left[K_{\lambda}\right]}\right)-v_{\xi}\left(a_{\xi}\right)$.

In our case, both $Y$ and $Y_{\lambda}(K)$ are lens spaces, hence L-spaces [36]. Therefore $H_{*}\left(B_{\xi}(Y, \bar{K})\right) \cong \mathbb{Z}$ for any $\xi \in \operatorname{Spin}^{c}(Y, K)$ and $H_{*}\left(\operatorname{Cone}\left(D_{\mathfrak{s}}\right)\right) \cong \mathbb{Z}$ for any $\mathfrak{s} \in$ $\operatorname{Spin}^{c}\left(Y_{\lambda}(K)\right)$ by Theorem 6.6. In fact for any field $\mathbb{F}, H_{*}\left(B_{\xi}(Y, \bar{K}) ; \mathbb{F}\right) \cong \mathbb{F}$ and $H_{*}\left(\operatorname{Cone}\left(D_{\mathfrak{s}}\right) ; \mathbb{F}\right) \cong \mathbb{F}$.

Lemma 6.7 After a possible change of orientation of the ambient manifold $Y$, we have $H_{*}\left(A_{\xi}(Y, \bar{K})\right) \cong \mathbb{Z}$ for any $\xi \in \underline{\operatorname{Spin}^{c}}(Y, K)$.

Proof This lemma is a slight generalisation of [38, Lemma 4.6]. It is only applied to a summand corresponding to the particular $\operatorname{Spin}^{c}$ structure $\mathfrak{s}$. Write the rational 
longitude of $K$ as $a \cdot \mu+p \cdot \lambda$ for some $a \in \mathbb{Z} \backslash\{0\}$. (Note that $Y_{\lambda}(K)$ is a lens space, so $\lambda$ cannot be the rational longitude.) By changing the orientation of $Y$ if necessary one can assume $a<0$.

The proof of Proposition 4.13 shows that $K^{\prime} \subset Y_{\lambda}(K)$ is primitive in $Y_{\lambda}(K)$. The map $G_{Y_{\lambda}(K), \bar{K}}$ is affinely modeled on the canonical projection: $\pi: \mathbb{Z} \rightarrow \mathbb{Z} / m$ where $m$ is the order of $H^{2}\left(Y_{\lambda}(K)\right)$ and $\pi$ is the map $i^{*}: H^{2}\left(Y_{\lambda}(K), K^{\prime}\right) \rightarrow H^{2}\left(Y_{\lambda}(K)\right)$ induced in cohomology; see [32, Section 2.2]. Therefore the groups

$$
\left(A_{\xi}\right)_{\xi \in G_{Y_{\lambda}(K), \bar{K}}^{-1}(\mathfrak{s})}
$$

form an affine copy of $m \cdot \mathbb{Z}$ in $\mathbb{Z} \cong \operatorname{Spin}^{c}(Y, K)$ and adding $\operatorname{PD}\left[K_{\lambda}\right]$ corresponds to a translation by $m$. By [32, Lemma 6.5] and the assumption $a<0$, for sufficiently large $n>n_{+}$the map $v_{\xi+n \cdot \operatorname{PD}\left[K_{\lambda}\right]}: A_{\xi+n \cdot \operatorname{PD}\left[K_{\lambda}\right]}(Y, \bar{K}) \rightarrow B_{\xi+n \cdot \operatorname{PD}\left[K_{\lambda}\right]}(Y, \bar{K})$ is an isomor-

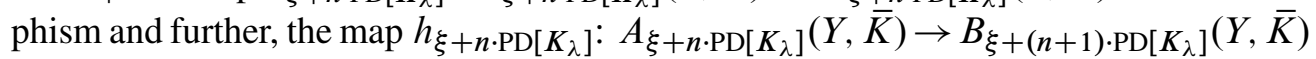
is trivial. If $n$ is sufficiently small, $n<n_{-}, v_{\xi+n \cdot \operatorname{PD}\left[K_{\lambda}\right]}$ is trivial and $h_{\xi+n \cdot \operatorname{PD}\left[K_{\lambda}\right]}$ is an isomorphism.

In general, the homology of the mapping cone of $D_{\mathfrak{s}}$ is an extension of $\operatorname{Ker}\left(\left(D_{\mathfrak{s}}\right)_{*}\right)$ by $\operatorname{Coker}\left(\left(D_{\mathfrak{s}}\right)_{*}\right)$ [47, Chapter 1]. Using homology with field $(\mathbb{F})$ coefficients, this extension splits: $h_{*}\left(\operatorname{Cone}\left(D_{\mathfrak{s}}\right)\right) \cong \operatorname{Ker}\left(\left(D_{\mathfrak{s}}\right)_{*}\right) \oplus \operatorname{Coker}\left(\left(D_{\mathfrak{s}}\right)_{*}\right)$. Another way to say this is $H_{*}\left(\operatorname{Cone}\left(D_{\mathfrak{s}}\right)\right) \cong H_{*}(\mathbb{X})$, where $\mathbb{X}$ is the short chain complex (see [38, Theorem 4.1])

$$
0 \longrightarrow H_{*}\left(\mathbb{A}_{\mathfrak{s}}(Y, \bar{K}) ; \mathbb{F}\right) \stackrel{\left(D_{\mathfrak{s}}\right)_{*}}{\longrightarrow} H_{*}\left(\mathbb{B}_{\mathfrak{s}}(Y, \bar{K}) ; \mathbb{F}\right) \longrightarrow 0 .
$$

Owing to the behavior of $D_{\mathfrak{s}} \mid A_{\xi+n \cdot \operatorname{PD}\left[K_{\lambda}\right]}(Y, \bar{K})$ for large, resp. small, $n$, the chain complex $\mathbb{X}$ splits into an infinite sum of acyclic subcomplexes

$$
\left.0 \rightarrow H_{*}\left(A_{\xi+n \cdot \operatorname{PD}\left[K_{\lambda}\right]}(Y, \bar{K}) ; \mathbb{F}\right) \stackrel{\left(h_{\xi+n} \cdot \operatorname{PD}\left[K_{\lambda}\right]\right.}{\cong}\right)_{*} H_{*}\left(B_{\xi+n \cdot \operatorname{PD}\left[K_{\lambda}\right]}(Y, \bar{K}) ; \mathbb{F}\right) \rightarrow 0
$$

for $n>n_{+}$,

$0 \rightarrow H_{*}\left(A_{\xi+n \cdot \operatorname{PD}\left[K_{\lambda}\right]}(Y, \bar{K}) ; \mathbb{F}\right) \underset{\left(v_{\xi+n} \cdot \operatorname{PD}\left[K_{\lambda}\right]\right)_{*}}{\cong} H_{*}\left(B_{\xi+(n+1) \cdot \operatorname{PD}\left[K_{\lambda}\right]}(Y, \bar{K}) ; \mathbb{F}\right) \rightarrow 0$

for $n<n_{-}$, and the nontrivial subcomplex between the groups $A_{\xi+n_{-} \cdot \operatorname{PD}\left[K_{\lambda}\right]}(Y, \bar{K})$ and $A_{\xi+n_{+} \cdot \mathrm{PD}\left[K_{\lambda}\right]}(Y, \bar{K})$ :
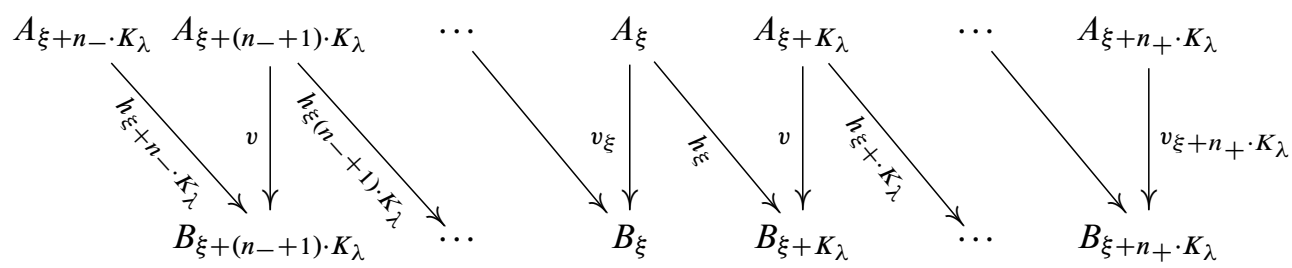
Since $H_{*}(\mathbb{X} ; \mathbb{F}) \cong H_{*}\left(B_{\xi+n \cdot \operatorname{PD}\left[K_{\lambda}\right]}(Y, \bar{K}) ; \mathbb{F}\right) \cong \mathbb{F}$,

$$
\operatorname{rank}\left(H_{*}\left(A_{\xi+n \cdot \operatorname{PD}\left[K_{\lambda}\right]}(Y, \bar{K}) ; \mathbb{F}\right)\right) \geq 1
$$

and the number of $A$ groups is one greater than that of $B$ groups, we have

$$
H_{*}\left(A_{\xi+n \cdot \operatorname{PD}\left[K_{\lambda}\right]}(Y, \bar{K}) ; \mathbb{F}\right) \cong \mathbb{F} .
$$

Since $\mathbb{F}$ was arbitrary, the universal coefficient theorem implies $H_{*}\left(A_{\xi}(Y, \bar{K})\right) \cong \mathbb{Z}$.

This phenomenon was studied by Ozsváth and Szabó [36] for knots in $S^{3}$. The result is purely algebraic, so it extends to our situation. As in the previous lemma, the proof is the same. The only change is that it is applied to a summand in the knot filtration corresponding to a fixed $\operatorname{Spin}^{c}$ structure on $Y$.

Lemma 6.8 [36, Lemmas 3.1 and 3.2] Under the conditions above, $\widehat{H F K}(Y, K, \xi)$ is either $\mathbb{Z}$ or 0 for any $\xi \in \underline{\operatorname{Spin}^{c}}(Y, K)$.

Proof Fix $\xi \in \operatorname{Spin}^{c}(Y, K)$. Lemmas 3.1 and 3.2 in [36] apply to a general $\mathbb{Z} \oplus \mathbb{Z}$ filtered chain complex $C$. We take $C$ to be $C_{\xi}$, notice that $C\{\max (i, j)=0\}$ corresponds to $A_{\xi}(Y, \bar{K})$ and $C\{\max (i, j-m)=0\}$ corresponds to $A_{\xi+m \cdot \operatorname{PD}[\mu]}(Y, \bar{K})$; in particular the hypotheses of the two lemmas are satisfied. One can therefore apply the argument in the proof of [36, Theorem 1.2] and the conclusion follows.

Juhasz [22] defined an Ozsváth-Szabó-type invariant called sutured Floer homology SFH for (balanced) sutured manifolds $(M, \gamma)$. (See also Ni [30].) One can construct a balanced sutured manifold $Y(K)$ starting from a knot $K$ by removing $N(K)$ and considering as sutures two copies of the meridian with opposite orientations. It is easy to see that $\operatorname{SFH}(Y(K)) \cong \widehat{H F K}(Y, K)$ by a natural identification between the corresponding chain complexes [22, Proposition 9.2].

The invariant $S F H$ also decomposes into different summands corresponding to $\operatorname{Spin}^{c}$ structures on $Y(K)$ which are in affine bijection with $H^{2}(Y(K), \partial Y(K))$, hence in bijection with $\operatorname{Spin}^{c}(Y, K)$. The isomorphism above preserves the splitting along

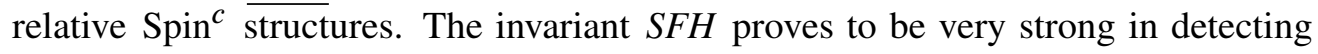
tautness and products:

Theorem 6.9 [23] Let $(M, \gamma)$ be an irreducible, balanced sutured manifold. Then $(M, \gamma)$ is taut if and only if $\operatorname{SFH}(M, \gamma) \neq 0$ and it is a product sutured manifold if and only if $\operatorname{SFH}(M, \gamma) \cong \mathbb{Z}$. 
The knot $K$ is rationally null-homologous and primitive in $Y$. Hence there is a surface $F$ properly embedded in $M=Y \backslash N(K)$, the exterior of $K$, whose boundary is the rational longitude of $K$. Without loss of generality we assume $F$ has minimal genus, $g$ say, among all such surfaces. One can cut open $M$ along $F$ and construct a sutured manifold $Y(F)$ whose suture is a parallel copy of $\partial F$. (See Gabai [11] for the original definitions of sutured manifolds and sutured manifold decompositions.) Our knot $K$ will be fibred if and only if $Y(F)$ is a product sutured manifold. One can compute $\operatorname{SFH}(Y(F))$ in terms of the knot Floer homology of $K$ via the surface decomposition theorem of Juhasz:

Theorem 6.10 [23, Theorem 1.3] Let $(M, \gamma)$ be a strongly balanced sutured manifold and $F$ a decomposing surface, and denote the manifold resulting from the decomposition by $(M(F), \gamma(F))$. Then

$$
\operatorname{SFH}(M(F), \gamma(F)) \cong \bigoplus_{\mathfrak{s} \in \operatorname{Out}(F)} \operatorname{SFH}(M, \mathfrak{s}),
$$

where $\operatorname{Out}(F)$ are the outer $\operatorname{Spin}^{c}$ structures on $(M, \gamma)$ with respect to $F$, ie the homology classes of vector fields in which one can find a representative which is never a negative multiple of the normal to $F$ with respect to some Riemannian metric on $M$ [23, Definition 1.1].

The strongly balanced hypothesis is a technical condition trivially satisfied in our case. The condition $\mathfrak{s} \in \operatorname{Out}(F)$ can be rephrased in terms of the Chern class of $\mathfrak{s}$ evaluated on $F: \mathfrak{s} \in \operatorname{Out}(F) \Longleftrightarrow\left\langle c_{1}\left(\mathfrak{s}, t_{0}\right),[F]\right\rangle=c\left(F, t_{0}\right)$, where $c\left(F, t_{0}\right)$ is a combinatorial quantity which in our case turns out to be $c\left(F, t_{0}\right)=1-2 g-p$ [23, Section 3]. See below for an explication of the terms in this formula.

The Chern class of a relative $\operatorname{Spin}^{c}$ structure $\mathfrak{s}$ is defined in the following way. Take a representative $v$ of $\mathfrak{s}$ (ie a nowhere vanishing vector field on $M$ with predetermined behaviour on $\partial M$ with respect to the sutures [23, Section 3]). Put a Riemannian metric on $M$ and consider the orientable 2-plane field $v^{\perp}$. Consider a trivialisation of $v_{\partial M}^{\perp}$ which exists because of the strongly balanced hypothesis. Then the Chern class of $\mathfrak{s}$ relative to this trivialisation is the obstruction to extending the trivialisation to all of $M$. (See [23] for details in the sutured case and [37] for the knot complement case.) There is a natural trivialisation $t_{0}$ on $\partial M$ to consider, namely the section consisting of vectors parallel to the meridian of $K$.

Since $H_{1}(M)$ contains no 2-torsion in our situation, the relative $\operatorname{Spin}^{c}$ structures on $M$ are identified by their Chern class (see [15] for the closed case; the relative case can be deduced by filling and applying the closed case result), which in turn 
are identified by the evaluation on the homology class $[F]$. Hence $\operatorname{Out}(F)$ consists precisely of one $\operatorname{Spin}^{c}$ structure $\xi_{0} \in \operatorname{Spin}^{c}(Y, K)$ (see the next paragraph for the exact identification of $\left.\xi_{\mathrm{o}}\right)$. Therefore, by Theorem 6.10, $\operatorname{SFH}(Y(F)) \cong \widehat{H F K}\left(Y, K, \xi_{\mathrm{o}}\right)$ which is 0 or $\mathbb{Z}$ by Lemma 6.8. As $K$ is primitive and $Y$ prime, $M$ is irreducible, and as $F$ is genus minimising, $Y(F)$ is taut. Thus $\operatorname{SFH}(Y(F)) \not 0$ by Theorem 6.9 and so must be isomorphic to $\mathbb{Z}$. Hence $K$ is fibred.

In fact, as for knots in $S^{3}$, one can identify $\operatorname{SFH}(Y(F))$ with the top summand with respect to the Alexander grading $\widehat{H F K}(Y, K, g+(p-1) / 2)$ [38, Section 3.7]: In [38] the Alexander grading $A$ on relative $\operatorname{Spin}^{c}$ structures is defined such that the Euler characteristic of the Floer homology is symmetric with respect to the origin. The same grading (after the identification $H^{2}(Y, K) \cong \mathbb{Z}$ given by declaring $[F]$ to be the positive generator) is defined in [31, Section 4.4] in terms of the Chern class of the $\operatorname{Spin}^{c}$ structures. By Juhasz's decomposition formula [23, Lemma 3.10 and Theorem 1.3], we get $\left\langle c_{1}\left(\xi_{0}, t_{0}\right),[F]\right\rangle=1-2 g-p$, hence $A\left(\xi_{0}\right)=(1-2 g-p) / 2$ and by conjugation invariance [31, Section 4.4 Equation 2], $\operatorname{SFH}(Y(F)) \cong \widehat{H F K}(Y, K, g+(p-1) / 2)$.

\subsection{Proof of Theorem 1.5}

We prove Theorem 1.5 here; it is an analogue of Ni's fibring theorem [30] in an orbifold setting. Recall that we have assumed that $K$ is a knot in an orbi-lens-space $\mathcal{L}$ which is primitive in $\mathcal{L}$ and which admits a nontrivial orbi-lens space surgery.

Proof of Theorem 1.5 When $\mathcal{L}$ is a manifold, this is just Theorem 6.5. Suppose then that $\mathcal{L}$ has a nonempty singular set, say $\mathcal{L}=\mathcal{L}(p, q ; a, b)$. Set $L_{0}=\mathcal{L}(p, q ; a, b) \backslash$ $N(\Sigma(\mathcal{L}(p, q ; a, b)))$ and note that as in the proof of Lemma 4.9,

$$
L_{0} \cong \begin{cases}S^{1} \times D^{2} & \text { if }|\Sigma(\mathcal{L}(p, q ; a, b))|=1, \\ S^{1} \times S^{1} \times[0,1] & \text { if }|\Sigma(\mathcal{L}(p, q ; a, b))|=2 .\end{cases}
$$

Since $K$ admits a nontrivial orbi-lens space surgery in $\mathcal{L}, L_{0}$ admits a nontrivial cosmetic surgery (cf the proof of Lemma 4.9). Proposition 5.1 then shows that $L_{0} \cong$ $S^{1} \times D^{2}$ (so we can suppose that $b=1$ ) and $K$ is a Berge-Gabai knot in $L_{0}$ (Definition 5.4). Let $n$ be the winding number of $K$ in $L_{0}$. Our hypotheses imply that $\operatorname{gcd}(p, n)=1$. Thus Theorem 6.1 implies that there is a locally trivial fibring of the exterior of $K$ in $L_{0}$ by surfaces which intersect $\partial L_{0}$ in curves parallel to the meridian slope of the solid torus $\overline{N(\Sigma(\mathcal{L}(p, q ; a)))}$. Therefore we can extend the fibration over the exterior of $K$ in $\mathcal{L}(p, q ; a)$ in such a way that it is everywhere transverse to $\Sigma(\mathcal{L}(p, q ; a))$. We endow each fibre $F$ of this surface fibration with the structure of a 2-orbifold by declaring each point of $F \cap \Sigma(\mathcal{L}(p, q ; a))$ to be a cone point of order $a$. In this way the exterior of $K$ in $\mathcal{L}(p, q ; a)$ admits an orbifold fibring with base the circle. 


\subsection{Proof of Theorem 1.7(1) and (2)}

Let $K$ be a hyperbolic knot without hidden symmetries such that $|\mathcal{C C}(K)|>1$. If $K$ is periodic, it is an unwrapped Berge-Gabai knot (Proposition 5.7) and so Corollary 6.2 implies that it is fibred. If $K$ is not periodic, then $Z(K)$ acts freely on $S^{3}$. Proposition 4.13 shows that the image $\bar{K}$ of $K$ in the lens space $\mathcal{L}_{K}$ admits a nontrivial lens space surgery. Since $\bar{K}$ is primitive in $\mathcal{L}_{K}$, Theorem 6.5 shows that $\bar{K}$, and therefore $K$, is fibred. Thus Theorem 1.7(1) holds. Part (2) of that theorem is an immediate consequence of the fibration result (1) and the fact that the knots are cyclically commensurable.

\section{Orientation reversing symmetries}

In this section we prove assertion (4) of Theorem 1.7.

Proposition 7.1 Let $K$ be an amphichiral hyperbolic knot. Then $|\mathcal{C C}(K)|=1$. Moreover, if $K$ has no hidden symmetry, then $|\mathcal{C}(K)|=1$.

Proof Let $K$ be an amphichiral knot with $S^{3} \backslash K \cong \mathbf{H}^{3} / \Gamma_{K}$. Fix an orientationreversing isometry $\theta: S^{3} \backslash K \rightarrow S^{3} \backslash K$ and lift it to $\tilde{\theta} \in \operatorname{Isom}\left(\mathbf{H}^{3}\right)$. Let $N\left(\Gamma_{K}\right)$ be the normalizer of $\Gamma_{K}$ in $\operatorname{Isom}\left(\mathbf{H}^{3}\right)$.

Then $\tilde{\theta} \in N\left(\Gamma_{K}\right)$ and normalizes $N^{+}\left(\Gamma_{K}\right)$. The action of $\tilde{\theta}$ permutes the index 2 subgroups of $N^{+}\left(\Gamma_{K}\right)$ and so it leaves invariant the unique such subgroup with a torus cusp (cf Lemma 4.3). Call this subgroup $\Gamma_{Z}$ and recall that $\mathbf{H}^{3} / \Gamma_{Z} \cong$ $\left(S^{3} \backslash K\right) / Z(K) \cong \mathcal{Z}_{K}$. Thus $\tilde{\theta}$ induces an orientation-reversing isometry $\bar{\theta}: \mathcal{Z}_{K} \rightarrow \mathcal{Z}_{K}$ which lifts to $\theta$.

Let $\mu_{K}, \lambda_{K}$ be a meridian, longitude basis of the first homology of the cusp of $S^{3} \backslash K$. It is clear that $\theta_{*}\left(\lambda_{K}\right)= \pm \lambda_{K}$ while $\theta_{*}\left(\mu_{K}\right)= \pm \mu_{K}$ by [19]. Projecting to $\mathcal{Z}_{K}$, we see that $\mu_{K} \mapsto \bar{\mu}$ and $\lambda_{K} \mapsto|Z(K)| \bar{\lambda}$ where $\bar{\mu}, \bar{\lambda}$ is a basis of the first homology of the cusp of $\mathcal{Z}_{K}$. Since $\bar{\theta}$ is orientation-reversing and lifts to $\theta$, there is an $\epsilon \in\{ \pm 1\}$ such that

$$
\bar{\theta}_{*}(\bar{\lambda})=\epsilon \bar{\lambda} \quad \text { and } \quad \bar{\theta}_{*}(\bar{\mu})=-\epsilon \bar{\mu} .
$$

It follows that, given any slope $\alpha=p \bar{\mu}+q \bar{\lambda}$ in the cusp of $\mathcal{Z}_{K}$,

$$
\Delta\left(\alpha, \bar{\theta}_{*}(\alpha)\right)=2|p q| \equiv 0(\bmod 2) .
$$

Since the set of slopes in the cusp of $\mathcal{Z}_{K}$ whose associated fillings yield orbi-lens spaces is invariant under $\bar{\theta}$ and the distance between any two such slopes is at most 1 
(cf the proof of Lemma 4.9), each such slope must be invariant under $\bar{\theta}$. But from the distance calculation immediately above, the only slopes invariant under $\bar{\theta}$ are those associated to $\bar{\mu}$ and $\bar{\lambda}$. The latter is the rational longitude of $\left|\mathcal{Z}_{K}\right|$ and so its associated filling cannot be an orbi-lens space. Thus the only slope which can yield an orbi-lens space is $r_{K}$, the slope associated to $\bar{\mu}$. Lemma 4.8 then shows that there is exactly one knot complement in the cyclic commensurability class of $S^{3} \backslash K$.

Proposition 7.1 together with Proposition 5.8 directly implies part (4) of Theorem 1.7.

Theorem 7.2 Let $S^{3} \backslash K$ be a chiral knot complement without hidden symmetries. Then $S^{3} \backslash K$ is not commensurable with an orbifold which admits an orientationreserving involution. That is, a knot complement without hidden symmetries in its orientable commensurator does not have hidden symmetries in its full commensurator.

Proof Suppose that $S^{3} \backslash K$ is commensurable with an orbifold $\mathcal{O}$ which admits an orientation-reversing involution. Let $\Gamma_{K}$ and $\Gamma_{\mathcal{O}}$ be discrete subgroups of PSL(2, $\mathbb{C}$ ) such that $\mathbf{H}^{3} / \Gamma_{K} \cong S^{3} \backslash K$ and $\mathbf{H}^{3} / \Gamma_{\mathcal{O}} \cong \mathcal{O}$. We furthermore suppose that $\Gamma_{\mathcal{O}}$ and $\Gamma_{K}$ intersect in a finite-index subgroup, by conjugating if necessary. By MostowPrasad rigidity, the involution of $\mathcal{O}$ corresponds to an element $g \in \operatorname{Isom}\left(\mathbf{H}^{3}\right)$ which conjugates the fundamental group of $\mathcal{O}$ in $\operatorname{PSL}(2, \mathbb{C})$ to itself. That is $g \Gamma_{\mathcal{O}} g^{-1}=\Gamma_{\mathcal{O}}$. Thus $g$ is contained in the full commensurator of $\Gamma_{\mathcal{O}}$, which is the same as the full commensurator of $\Gamma_{K}$. This implies that $\Gamma_{K}$ is commensurable with $g \Gamma_{k} g^{-1}$, or that $S^{3} \backslash K$ is commensurable with its image under an orientation-reversing involution. But this knot complement has the same volume, which contradicts Proposition 5.8.

\section{References}

[1] I R Aitchison, J H Rubinstein, Combinatorial cubings, cusps, and the dodecahedral knots, from: "Topology '90 (Columbus, OH, 1990)", (B Apanasov, W D Neumann, A W Reid, L Siebenmann, editors), Ohio State Univ. Math. Res. Inst. Publ. 1, de Gruyter, Berlin (1992) 17-26 MR1184399

[2] I R Aitchison, J H Rubinstein, Geodesic surfaces in knot complements, Experiment. Math. 6 (1997) 137-150 MR1474574

[3] M A Armstrong, The fundamental group of the orbit space of a discontinuous group, Proc. Cambridge Philos. Soc. 64 (1968) 299-301 MR0221488

[4] J Berge, The knots in $D^{2} \times S^{1}$ which have nontrivial Dehn surgeries that yield $D^{2} \times S^{1}$, Topology Appl. 38 (1991) 1-19 MR1093862

[5] M Boileau, B Leeb, J Porti, Geometrization of 3-dimensional orbifolds, Ann. of Math. 162 (2005) 195-290 MR2178962 
[6] F Bonahon, J-P Otal, Scindements de Heegaard des espaces lenticulaires, Ann. Sci. École Norm. Sup. 16 (1983) 451-466 MR740078

[7] D Boyd, The A-polynomials of families of symmetric knots, Lecture notes, PIMS-MSRI conference on Knots and Manifolds (University of British Columbia, Vancouver) (2004) Available at http://www.math.ubc.ca/ boyd/Apoly.symm.pdf

[8] KS Brown, Trees, valuations, and the Bieri-Neumann-Strebel invariant, Invent. Math. 90 (1987) 479-504 MR914847

[9] D Calegari, N M Dunfield, Commensurability of 1-cusped hyperbolic 3-manifolds, Trans. Amer. Math. Soc. 354 (2002) 2955-2969 MR1895211

[10] M Culler, C M Gordon, J Luecke, P B Shalen, Dehn surgery on knots, Ann. of Math. 125 (1987) 237-300 MR881270

[11] D Gabai, Foliations and the topology of 3-manifolds, J. Differential Geom. 18 (1983) 445-503 MR723813

[12] D Gabai, Foliations and the topology of 3-manifolds. II, J. Differential Geom. 26 (1987) 461-478 MR910017

[13] D Gabai, Surgery on knots in solid tori, Topology 28 (1989) 1-6 MR991095

[14] D Gabai, 1-bridge braids in solid tori, Topology Appl. 37 (1990) 221-235 MR1082933

[15] R E Gompf, Spin ${ }^{c}$-structures and homotopy equivalences, Geom. Topol. 1 (1997) 41-50 MR1475553

[16] F González-Acuña, W C Whitten, Imbeddings of three-manifold groups, Mem. Amer. Math. Soc. 99, no. 474, Amer. Math. Soc. (1992) MR1117167

[17] O Goodman, D Heard, C Hodgson, Commensurators of cusped hyperbolic manifolds, Experiment. Math. 17 (2008) 283-306 MR2455701

[18] C M Gordon, Dehn surgery and satellite knots, Trans. Amer. Math. Soc. 275 (1983) 687-708 MR682725

[19] C M Gordon, J Luecke, Knots are determined by their complements, J. Amer. Math. Soc. 2 (1989) 371-415 MR965210

[20] A Hatcher, Notes on basic 3-manifold Topology, online book (2007) Available at http://www . math. cornell.edu/ hatcher/3M/3Mdownloads.html

[21] N Hoffman, Commensurability classes containing three knot complements, Algebr. Geom. Topol. 10 (2010) 663-677 MR2606796

[22] A Juhász, Holomorphic discs and sutured manifolds, Algebr. Geom. Topol. 6 (2006) 1429-1457 MR2253454

[23] A Juhász, Floer homology and surface decompositions, Geom. Topol. 12 (2008) 299350 MR2390347 
[24] S Kojima, Isometry transformations of hyperbolic 3-manifolds, Topology Appl. 29 (1988) 297-307 MR953960

[25] P B Kronheimer, T S Mrowka, Dehn surgery, the fundamental group and SU(2), Math. Res. Lett. 11 (2004) 741-754 MR2106239

[26] M L Macasieb, T W Mattman, Commensurability classes of $(-2,3, n)$ pretzel knot complements, Algebr. Geom. Topol. 8 (2008) 1833-1853 MR2448875

[27] J Morgan, G Tian, Ricci flow and the Poincaré conjecture, Clay Math. Monogr. 3, Amer. Math. Soc. (2007) MR2334563

[28] W D Neumann, Kleinian groups generated by rotations, from: “Groups-Korea '94 (Pusan)", (A C Kim, D L Johnson, editors), de Gruyter, Berlin (1995) 251-256 MR1476967

[29] W D Neumann, A W Reid, Arithmetic of hyperbolic manifolds, from: "Topology '90 (Columbus, OH, 1990)", (B Apanasov, W D Neumann, A W Reid, L Siebenmann, editors), Ohio State Univ. Math. Res. Inst. Publ. 1, de Gruyter, Berlin (1992) 273-310 MR1184416

[30] Y Ni, Knot Floer homology detects fibred knots, Invent. Math. 170 (2007) 577-608 MR2357503

[31] Y Ni, Link Floer homology detects the Thurston norm, Geom. Topol. 13 (2009) 29913019 MR2546619

[32] P Ozsváth, Z Szabó, Knot Floer homology and rational surgeries arXiv: math.GT/0504404

[33] P Ozsváth, Z Szabó, Holomorphic disks and knot invariants, Adv. Math. 186 (2004) 58-116 MR2065507

[34] P Ozsváth, Z Szabó, Holomorphic disks and three-manifold invariants: properties and applications, Ann. of Math. 159 (2004) 1159-1245 MR2113020

[35] P Ozsváth, Z Szabó, Holomorphic disks and topological invariants for closed threemanifolds, Ann. of Math. 159 (2004) 1027-1158 MR2113019

[36] P Ozsváth, Z Szabó, On knot Floer homology and lens space surgeries, Topology 44 (2005) 1281-1300 MR2168576

[37] P Ozsváth, Z Szabó, Holomorphic disks, link invariants and the multi-variable Alexander polynomial, Algebr. Geom. Topol. 8 (2008) 615-692 MR2443092

[38] J Rasmussen, Lens space surgeries and L-space homology spheres arXiv: $0710.2531 \mathrm{v} 1$

[39] A W Reid, Arithmeticity of knot complements, J. London Math. Soc. 43 (1991) 171-184 MR1099096

[40] A W Reid, G S Walsh, Commensurability classes of 2-bridge knot complements, Algebr. Geom. Topol. 8 (2008) 1031-1057 MR2443107 
[41] T Sakai, Geodesic knots in a hyperbolic 3-manifold, Kobe J. Math. 8 (1991) 81-87 MR1134707

[42] R E Schwartz, The quasi-isometry classification of rank one lattices, Inst. Hautes Études Sci. Publ. Math. (1995) 133-168 MR1383215

[43] J Stallings, On fibering certain 3-manifolds, from: "Topology of 3-manifolds and related topics (Proc. The Univ. of Georgia Institute, 1961)”, (M K Fort, editor), PrenticeHall, Englewood Cliffs, N.J. (1962) 95-100 MR0158375

[44] J Stallings, Constructions of fibred knots and links, from: "Algebraic and geometric topology (Proc. Sympos. Pure Math., Stanford Univ., Stanford, CA, 1976), Part 2”, (R J Milgram, editor), Proc. Sympos. Pure Math. XXXII, Amer. Math. Soc. (1978) 55-60 MR520522

[45] W P Thurston, A norm for the homology of 3-manifolds, Mem. Amer. Math. Soc. 59, no. 339, Amer. Math. Soc. (1986) MR823443

[46] V Turaev, Torsion invariants of $\operatorname{Spin}^{c}$-structures on 3-manifolds, Math. Res. Lett. 4 (1997) 679-695 MR1484699

[47] C A Weibel, An introduction to homological algebra, Cambridge Studies in Adv. Math. 38, Cambridge Univ. Press (1994) MR1269324

MB: Institut de Mathématiques de Toulouse, Université Paul Sabatier 118 route de Narbonne, F-31062 Toulouse Cedex 9, France

SB, RC: Département de mathématiques, Université du Québec à Montréal PO Box 8888, Centre-ville, Montréal QC H3C 3P8, Canada

GSW: Department of Mathematics, Tufts University

503 Boston Ave, Medford MA 02155, USA

boileau@picard.ups-tlse.fr, boyer.steven@uqam.ca, radu.cebanu@cirget.ca, genevieve.walsh@tufts.edu

http://picard.ups-tlse.fr/homepage/boileau.html, http://www . cirget. uqam.ca/boyer/boyer.html, http://www.tufts.edu/ gwalsh01/

Proposed: Cameron Gordon

Received: 8 February 2011

Seconded: Walter Neumann, Colin Rourke Accepted: 27 November 2011 